\title{
Consenso del implante valvular aórtico percutáneo del Colegio Argentino de Cardioangiólogos Intervencionistas 2016
}

\author{
Consensus on Percutaneous Aortic Valve Implantation - CACl 2016
}

\begin{abstract}
${ }^{1}$ Coordinadora: Dra. Carla Agatiello. Comité de Redacción: Dra. Alfonsina Candiello, Dr. Matías Sztejfman, Dr. Carlos Fava, Dr. Diego Grinfeld, Dr. Ignacio Seropian, Dr. Antonio Scuteri, Dr. Aníbal Damonte, Dr. Juan Mieres. Comité de Revisión: Dr. Hugo Londero, Dr. Oscar Mendiz, Dr. Fernando Cura, Dr. Daniel Berrocal, Dr. Miguel Payaslian, Dr. Alfredo Rodríguez, Dr. José Álvarez, Dr. Claudio Cigalini, Dr. Ernesto Torresani, Dr. Arturo Fernández Murga, Dr. José Gómez Moreno, Dr. Marcelo Menéndez, Dr. Alejandro Cherro, Dr. Jorge Baccaro, Dr. Alejandro Álvarez
\end{abstract}

Revista Argentina de Cardioangiología Intervencionista 2016;7(4):194-210

INDICE

1. Introducción y estado actual

2. Indicaciones y contraindicaciones

2.1 Heart Team

2.2 Indicaciones

2.3 Contraindicaciones

2.4 Valvulopatía aórtica

3. Tipos de prótesis

3.1 Autoexpandibles

3.1.1 CoreValve (Medtronic)

3.1.2 ACURATE (Symetis)

3.1.3 Lotus Valve (Boston Scientific)

3.1.4 JenaValve (JenaValve Technology)

3.1.5 Portico Valve System (St. Jude Medical)

3.2 Balón expandible

3.2.1 Edwards Sapien (Edwards Lifesciences)

4. Elección del tamaño de la prótesis

4.1 Mediciones del anillo valvular y sus estructuras adyacentes

4.2 Estudio de accesos vasculares

\section{Accesos}

5.1 Tipos de accesos

5.2 Resultados de los accesos

\section{Complicaciones}

6.1 Leak paravalvular

6.2 Bloqueo auriculoventricular

6.3 Complicaciones del acceso

6.4 Stroke

7. Cuidados posimplante

7.1 Terapia antitrombótica durante el TAVI

7.2 Antiagregación luego del implante de TAVI

7.3 Anticoagulación

7.4 Fibrilación auricular

7.5 Seguimiento

8. Situaciones especiales

8.1 Válvula bicúspide

8.2 Insuficiencia aórtica pura

8.3 Valve-in-valve

8.4 Riesgo intermedio y bajo riesgo quirúrgico.

\section{INTRODUCCIÓN}

La estenosis aórtica severa degenerativa prevalece en adultos mayores, por lo que su número se encuentra en aumento a raíz del envejecimiento de la población. Se define como estenosis aórtica severa cuando el área valvular es menor de $1 \mathrm{~cm}^{2}$ o de $0,6 \mathrm{~cm}^{2} / \mathrm{m}^{2}$ de superficie corporal. Su prevalencia es del 4,6\% de los adultos mayores de 75 años ${ }^{1}$. Dado que el tratamiento médico no modifica la evolución natural de la enfermedad, el tratamiento definitivo consiste en el reemplazo valvular aórtico. La cirugía de reemplazo valvular con circulación extracorpórea es el tratamiento habitual. Sin embargo, en pacientes muy añosos y/o con comorbilidades el tratamiento quirúrgico puede presentar una muy alta mor- 
talidad, por lo que en los últimos años ha surgido el implante valvular aórtico percutáneo (TAVI) como alternativa de la cirugía en este tipo de pacientes.

La valvuloplastia aórtica con balón (BAV) en forma percutánea puede ser utilizada como un tratamiento paliativo en pacientes que no son candidatos a cirugía ni a TAVI, o como puente al tratamiento definitivo. Sin embargo, sus indicaciones y contraindicaciones no serán tratadas en el presente documento. Las indicaciones de tratamiento definitivo (cirugía o TAVI) de la estenosis valvular aórtica se encuentran detalladas en consensos nacionales ${ }^{2,3}$ e internacionales ${ }^{4,5}$.

El primer TAVI fue realizado por el Profesor francés Alain Cribier en el año 2002. En la Argentina, la técnica del TAVI fue realizada en el año 2009 y hasta la fecha se han realizado más de 1500 procedimientos en más de 50 centros con diferentes grados de penetración. Sin embargo, no existen documentos nacionales y son pocos los consensos internacionales ${ }^{6,7}$.

A modo de educación continua, el CACI propuso realizar un consenso TAVI para permitir ser un instrumento de formación desde los centros con más experiencia a los de menos experiencia o a aquellos que quisieran iniciarse en la técnica. Es por ello que se convocó a centros de alto volumen TAVI (para nuestro medio, $>100$ y 50-100 TAVI implantadas) para colaborar en la elaboración de este documento.

El objetivo principal de este consenso es elaborar recomendaciones basadas en la evidencia disponible y la experiencia de los centros de mayor experiencia en la Argentina.

\section{INDICACIONES Y CONTRAINDICACIONES}

\subsection{Heart team o Equipo del corazón}

La expectativa de vida se encuentra en ascenso, lo que conlleva un aumento de las morbilidades, haciendo difícil la toma de decisiones. Los pacientes con estenosis aórtica severa presentan edad avanzada, lo que se asocia a una alta tasa de complicaciones y elevada mortalidad. Por este motivo el concepto del "equipo de corazón" o "heart team" (HT) se torna fundamental a la hora de la evaluación y la toma de decisiones. El concepto del HT es de un grupo multidisciplinario y colaborativo en donde cada uno de los integrantes debe aportar su opinión en libertad sin generarse conflictos internos. Debe estar formado por cardiólogos, intervencionistas con experiencia en patología estructural, cirujanos cardiovasculares, especialistas en imágenes (ecocardiografía, TAC, RMN), anestesistas y enfermeros. De acuerdo a las condiciones de los pacientes, puede ser necesario sumar neurólogos, clínicos, oncólogos, gerontólogos, psiquiatras o psicólogos. El número de pacientes muy añosos se encuentra en ascenso, y estos presentan mayor morbilidad y fragilidad. Esto hace necesario que sean evaluados por un especialista en gerontología. Si bien no existe ningún score desarrollado para TAVI en particular, se pueden utilizar entre ellos el Multidimensional Geriatric Assesment ${ }^{8}$, el ISAR o el SHERPA', que no sólo incluyen los scores de riesgos como el STS y el EuroSCORE, sino que agregan test cognitivos, score de fragilidad, estado de nutrición, actividades diarias y movilidad. Estos test se relacionan con eventos cardiovasculares mayores y mortalidad al mes y al año.

El objetivo del HT es optimizar la evaluación de riesgo y definir cuál es la mejor estrategia, además de comunicar claramente y responder todas las preguntas del paciente y la familia con respecto a los riesgos, expectativas y calidad de vida futura. Los hospitales que deseen contar con la posibilidad de realizar implantes valvulares deben contar con un HT entrenado, que pueda evaluar en forma correcta y discriminar el riesgo, ofreciendo la estrategia (cirugía, endovascular o tratamiento paliativo) conveniente para cada paciente. Tiene además la obligación de participar en diferentes registros o estudios, actividades académicas y publicar sus resultados en forma completa. De esta forma el HT brinda eficacia en la toma de decisiones y ahorra costos a los sistemas de salud.

\section{Recomendaciones $^{5}$}

\section{Clase I}

Todo paciente con enfermedad valvular severa debe ser evaluado por un heart team multidisciplinario cuando es considerado a una intervención (Nivel de Evidencia: C).

\section{Clase IIa}

Es razonable consultar o referir a un centro con heart team para definir las opciones de tratamiento en pacientes con enfermedad valvular aórtica severa por: 1 ) ausencia de síntomas, 2) pacientes con múltiples comorbilidades que se considere que requieran intervención valvular (Nivel de Evidencia: C).

\subsection{Indicaciones TAVI}

El reemplazo valvular aórtico mediante cirugía continúa siendo el tratamiento de elección para la estenosis aórtica. Sin embargo, en las siguientes situaciones se recomienda el TAVI.

\section{Recomendaciones}

\section{Clase I}

TAVI está recomendado en pacientes que cumplen criterios para reemplazo de válvula aórtica con contraindicación para la cirugía de reemplazo valvular aórtico con una sobrevida predictiva mayor a 12 meses (Nivel de Evidencia: B) ${ }^{10,11}$.

\section{Clase IIa}

TAVI es una alternativa razonable a la cirugía en pacientes con criterios de reemplazo valvular aórtico con alto riesgo quirúrgico para la cirugía de reemplazo valvular aórtico (Nivel de Evidencia: B) ${ }^{12,13}$.

\section{Clase III: sin beneficio}

TAVI no está recomendado en pacientes que presenten comorbilidades que impedirían el beneficio esperado de la corrección de la estenosis aórtica ${ }^{10}$. 


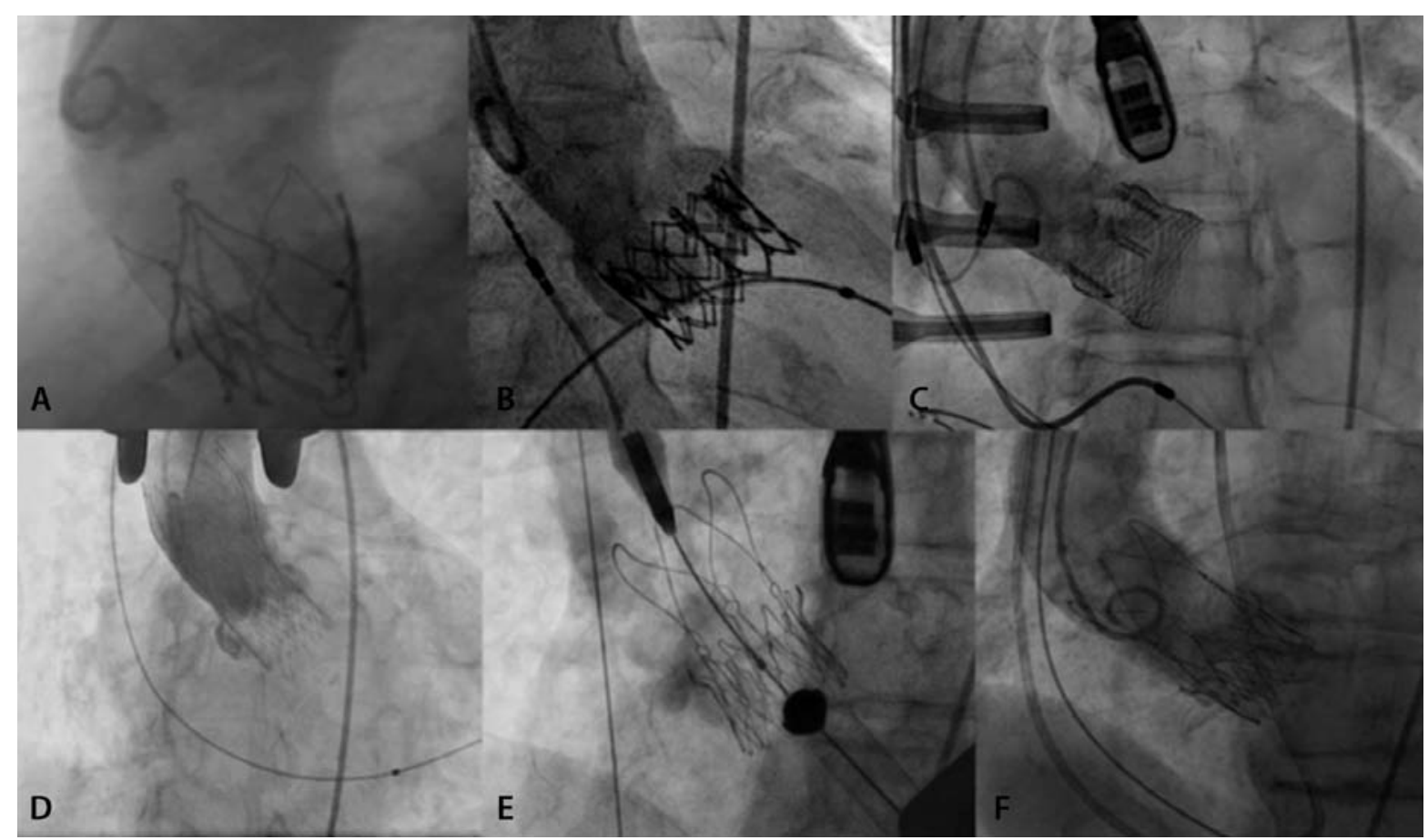

Figura 1. Prótesis disponibles para TAVI en Argentina. A: JenaValve. B: SAPIEN XT. C: Lotus. D: CoreValve. E: ACURATE TA. F: ACURATE neo.

\subsection{Contraindicaciones TAVI}

\section{Absolutas}

- Ausencia de heart team.

- Ausencia de cirugía cardíaca en el centro.

- Idoneidad para realizar TAVI no confirmada por el heart team.

- Expectativa de vida $<1$ año.

- Mejoría de la calidad de vida post-TAVI poco probable por las comorbilidades.

- Severo compromiso primario de otra enfermedad valvular con mayor contribución de los síntomas del paciente que pueden ser tratados solamente por cirugía.

- Anillo inadecuado (<18 mm o > al diámetro máximo recomendado por la prótesis a implantar).

- Trombo en el ventrículo izquierdo.

- Endocarditis activa.

- Placas con trombo móvil en aorta ascendente o en el arco aórtico, que no revierten con el tratamiento médico.

\section{Relativas}

- Válvulas bicúspides o no calcificadas.

- Inestabilidad hemodinámica.

- Fracción de eyección $<20 \%$.

- Acceso apical: enfermedad pulmonar severa, ápex cardíaco no accesible.

\subsection{Valvuloplastia aórtica}

La valvuloplastia aórtica con balón se utiliza en pacientes inestables como puente al TAVI o a la cirugía, y en aquellos pacientes que por sus comorbilidades no son pasibles de reemplazo valvular aórtico o TAVI. También puede ser utilizada en pacientes que requieren una cirugía no cardíaca y presentan indicación de trata- miento de estenosis aórtica severa (Clase IIa; Nivel de Evidencia: $\mathrm{C})^{5}$.

\section{TIPOS DE PRÓTESIS}

Existen variados dispositivos para el tratamiento de la estenosis aórtica severa mediante TAVI (Figura 1). Estas pueden clasificarse de diversas maneras:

Existen válvulas de pericardio porcino y de pericardio bovino, autoexpandibles y balón expandible, con sistema de liberación con mayor o menor control y distintos mecanismos y técnicas de implante (Tabla 1).

\subsection{Autoexpandibles}

\subsubsection{CoreValve (Medtronic)}

Dispositivo autoexpandible de níquel-titanio (Nitinol) con valvas de pericardio porcino, en diámetros de 23 a $31 \mathrm{~mm}$ para anillos aórticos de 18 a $29 \mathrm{~mm}$ de diámetro. De acceso retrógrado (transfemoral, transaórtico, transubclavio o transcarotídeo). Por su arquitectura singular, CoreValve combina un marco de nitinol con un diseño de válvula bioprotésica para reducir la tensión en las valvas de la válvula y maximizar la coaptación. La posición supraanular de los velos de CoreValve optimiza el área y el flujo mientras que preserva la forma circular y una coaptación óptima. Perfil de cruce bajo, compatible con introductor de $18 \mathrm{Fr}$ en todos los tamaños de válvula. Permite cuerdas guía de 0,035 ”. La versión CoreValve Evolute R, de segunda generación, se diferencia de la CoreValve porque es recuperable, reposicionable, con un dispositivo de liberación con mayor control y presenta en una estructura de stent 
TABLA 1. Principales características de cada bioprótesis.

\begin{tabular}{|c|c|c|c|c|c|c|c|c|}
\hline & CoreValve & $\begin{array}{l}\text { CoreValve } \\
\text { Evolute R }\end{array}$ & ACURATE neo & ACURATE TA & Lotus & JenaValve & SAPIEN XT & SAPIEN 3 \\
\hline $\begin{array}{l}\text { Mecanismo } \\
\text { de expansión }\end{array}$ & Autoexpandible & Autoexpandible & Autoexpandible & Autoexpandible & $\begin{array}{l}\text { Mecánicamente } \\
\text { expandible }\end{array}$ & Autoexpandible & $\begin{array}{l}\text { Balón } \\
\text { expandible }\end{array}$ & $\begin{array}{l}\text { Balón } \\
\text { expandible }\end{array}$ \\
\hline Stent & Nitinol & Nitinol & Nitinol & Nitinol & Nitinol & Nitinol & Cromocobalto & Cromocobalto \\
\hline Material valvas & Porcino & Porcino & Porcino & Porcino & Bovino & Porcino & Bovino & Bovino \\
\hline Medidas & 23 a $31 \mathrm{~mm}$ & 23 a $29 \mathrm{~mm}$ & S,M,L 21 a $27 \mathrm{~mm}$ & $\mathrm{~S}, \mathrm{M}, \mathrm{L} 21$ a $27 \mathrm{~mm}$ & 23 a $27 \mathrm{~mm}$ & 23 a $27 \mathrm{~mm}$ & 23 a $29 \mathrm{~mm}$ & 23 a $29 \mathrm{~mm}$ \\
\hline Introductor & $18 \mathrm{~F}(22 \mathrm{FOD})$ & $14 \mathrm{~F}$ (18 F OD) & $18 \mathrm{~F}$ & $33 F$ & $20-22 \mathrm{~F}(22-24 \mathrm{FOD})$ & $32 \mathrm{~F}$ & $16-20 \mathrm{~F}$ & $14-16 \mathrm{~F}$ \\
\hline Acceso & Femoral & Femoral & Femoral & Apical & Femoral & Apical & $\begin{array}{l}\text { Femoraly } \\
\text { apical }\end{array}$ & $\begin{array}{l}\text { Femoral y } \\
\text { apical }\end{array}$ \\
\hline
\end{tabular}

S,M,L: small, medium, large. F: french; OD: diámetro externo (outer diameter)

más corto y de menor diámetro de cruce, con una pollera extendida para mejorar la aposición de la prótesis y disminuir las fugas paravalvulares. Sus diámetros van de 23 a 29 mm, para anillos valvulares aórticos de 18 a $27 \mathrm{~mm}$ de diámetro. El sistema de liberación es de 14 french $(\mathrm{Fr})$, para cuerdas guía $0,035^{\prime}$.

\subsubsection{ACURATE (Symetis)}

Dispositivo autoexpandible de nitinol. De acceso retrógrado transfemoral (ACURATE neo/TF) o anterógrado transapical (ACURATE TA).

La bioprótesis aórtica ACURATE neo se utiliza en combinación con el sistema de liberación transfemoral ACURATE TF. Combina un stent autoexpandible de nitinol con una válvula porcina biológica. Está disponible en tres tamaños diferentes: $S, M$ y L, para anillo aórticos de 21 a $27 \mathrm{~mm}$ de diámetro. Esta válvula tiene un exclusivo sistema de liberación top-down (de arriba hacia abajo), a favor del flujo sanguíneo, lo que permite mayor estabilidad en la liberación, menos turbulencia y menos saltos durante el posicionamiento de la prótesis. El sistema de liberación posee una longitud útil de $109 \mathrm{~cm}$, es compatible con introductor de 18 french y con cuerda guía de 0,035 pulgadas.

La bioprótesis aórtica transapical ACURATE TA está diseñada para su uso con el sistema de suministro transapical ACURATE TA. Este dispositivo transapical combina un stent de nitinol autoexpandible con una válvula biológica porcina. La válvula ACURATE TA está disponible en los tres tamaños siguientes: $S, M$ y L, para anillos de 21 a $27 \mathrm{~mm}$ de diámetro. El sistema de liberación tiene un perfil de cruce de 33 french, una longitud útil de $34 \mathrm{~cm}$ y es compatible con cuerdas de 0,035 ".

\subsubsection{Lotus Valve (Boston Scientific)}

Válvula mecánicamente expandible de nitinol, con valvas de pericardio bovino, recubierta por una membrana de poliuretano flexible para disminuir el leak perivalvular. De acceso retrógrado. Es un dispositivo reposicionable y recapturable. Esta válvula no necesita de armado en la sala, viene premontada. Se encuentra disponible en medidas de 23 a $27 \mathrm{~mm}$ para anillo valvulares de 20 a $27 \mathrm{~mm}$ de diámetro. El sistema de liberación es compatible con introductor de 14 french y se utilizan cuerdas de 0,035 ”. El sistema para la válvula $\mathrm{N}^{\circ} 23$ es de $148 \mathrm{~cm}$ de largo y para las 25 y 27 de $166 \mathrm{~cm}$ de longitud. La válvu- la Lotus de $23 \mathrm{~mm}$ es compatible con el introductor Lotus small y las de 25 y 27 con introductor Lotus large. En nuestro país la empresa Boston Scientific entrega el kit de implante con la válvula montada en el sistema de liberación, introductor femoral Lotus y cuerda preformada Safari. No es necesario marcapasos y ritmo ventricular rápido durante su posicionamiento y liberación.

\subsubsection{JenaValve (JenaValve Technology)}

Autoexpandible. Sólo para acceso anterógrado transapical. La prótesis JenaValve utiliza una válvula de raíz aórtica porcina unida a un stent autoexpandible de nitinol. No es necesario marcapasos y ritmo ventricular rápido durante su posicionamiento y despliegue. La JenaValve se presenta en medidas de 23, 25 y $27 \mathrm{~mm}$, para anillos nativos de 21 a $27 \mathrm{~mm}$ de diámetro. El sistema de liberación utiliza un introductor de 32 french de acceso apical, para cuerda de 0,035 ".

\subsubsection{Portico Valve System (St. Jude Medical)}

Dispositivo de pericardio porcino montado sobre stent de nitinol autoexpandible. De acceso retrógrado. Esta válvula es reposicionable y recuperable. Este dispositivo se encuentra disponible en diámetros de 23 a 29 $\mathrm{mm}$ para anillos aórticos nativos de 19 a $27 \mathrm{~mm}$ de diámetro. El sistema de liberación es compatible con introductor de 18 french en las válvulas de 23 y $25 \mathrm{~mm}$ y de 19 french en las de 27 y $29 \mathrm{~mm}$.

\subsection{Balón expandible}

\subsubsection{Edwards SAPIEN (Edwards Lifesciences)}

Realizada en cromo cobalto y con valvas de pericardio bovino. Única bioprótesis valvular aórtica percutánea balón expandible de acceso retrógrado o transapical. La versión SAPIEN XT se encuentra disponible en medidas de 23 a $29 \mathrm{~mm}$ para anillo valvulares de 18 a $27 \mathrm{~mm}$ de diámetro. El dispositivo de liberación es compatible con introductores de 16 a 20 french y cuerda guía de $0,035^{\prime}$. La versión más moderna SAPIEN 3 se diferencia de su antecesora en el agregado de una cubierta de tela externa (pollera) para disminuir el leak paravalvular y de poseer struts más delgados. Se encuentra disponible en diámetros de 20 a $29 \mathrm{~mm}$ para anillo valvulares de 18 a $29 \mathrm{~mm}$ de diámetro. El sistema de liberación es deflectable y compatible con intro- 


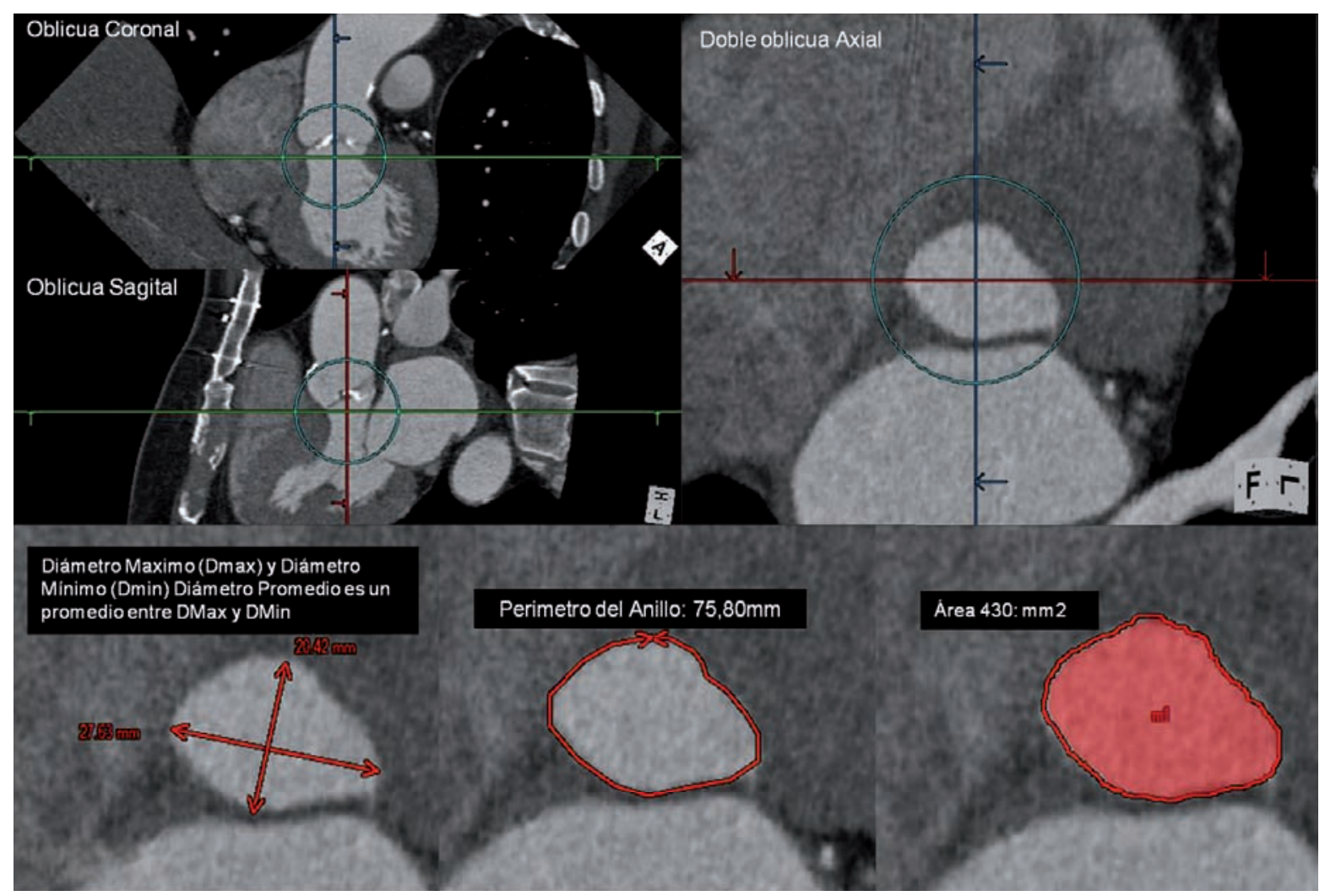

Figura 2. Mediciones del anillo valvular mediante angio-TC.

ductor de 14 french en las válvulas 20 a $26 \mathrm{~mm}$ y con introductor de 16 french para las de $29 \mathrm{~mm}$. Siempre con cuerda guía de $0,035 "$

\section{ELECCIÓN DEL TAMAÑO DE LA PRÓTESIS}

\subsection{Mediciones del anillo valvular y sus estructuras adyacentes}

El anillo aórtico para los propósitos del TAVI es la circunferencia virtual formada por la unión de los puntos más basales de las tres valvas de la válvula aórtica (VA). Inmediatamente por debajo de él se encuentra el tracto de salida del ventrículo izquierdo (TSVI) e inmediatamente por arriba el seno de la raíz de aorta.

El análisis del anillo aórtico por métodos de imagen en 3 dimensiones (3D) ha mostrado que no se trata de una estructura meramente circular, sino que por el contrario es generalmente de tipo ovoide, siendo el diámetro máximo aproximadamente un 30\% más largo que el diámetro mínimo ${ }^{14}$. De esta forma, los métodos que emplean imágenes en 2 dimensiones, como la angiografía de la raíz aórtica, la ecografía transtorácica (ETT) o la ecografía transesofágica (ETE), pueden generar errores a la hora de medir este tipo de estructuras ya que la miden en un solo plano. El uso de ETE en 3D ha demostrado tener buena correlación entre este y la angio-TC pero a la hora de analizar anillos con gran infiltración cálcica, situación frecuente en esta patología, la definición es un poco menor.
El uso de tecnología que permite la reconstrucción en 3D del anillo ha mostrado mejor entendimiento de esta estructura y mayor exactitud a la hora de medirla. De esta manera, la selección de la medida de prótesis a usar es más precisa y disminuye notablemente los leaks paravalvulares por falta de aposición de la misma con el anillo $o^{15}$. Para una adecuada medición del anillo, lo ideal es solicitar una angiotomografía multicorte (angio-TC) de al menos 64 filas, gatillada por ECG, con fases cardíaca 30-40\%, con cortes cada 0,5 a $1 \mathrm{~mm}$. Una vez adquirido el estudio debemos posprocesarlo en un software de manejo de imágenes DICOM (Ónix, Osirix, Horos, etc.) para obtener la imagen adecuada donde realizar las mediciones. Se debe realizar una reconstrucción multiplanar de la angio-TC para obtener tres tipos de cortes: corte coronal, corte sagital y corte doble oblicuo axial. Para la medida del anillo usaremos la fase sistólica 30-40\% del intervalo R-R, donde las valvas de la VA están bien abiertas, similar que en el ETE. El paso crítico para realizar mediciones adecuadas consiste en alinear las tres valvas de la VA en un mismo plano para de esta manera obtener una imagen real del anillo, sin distorsiones, en el corte doble oblicua axial ${ }^{16}$. En este corte se realizan las mediciones de diámetro máximo, diámetro mínimo, diámetro promedio, perímetro y área (Figura 2). También se puede medir el anillo en oblicua coronal y oblicua sagital, pero esta medición no será la más adecuada ya que se lo estaría evaluando en un solo plano y ambas mediciones pueden tener algún grado de diferencia ${ }^{17}$ (Figura 3). Los fabricantes de válvulas ofrecen información acerca de qué tamaño de válvu- 


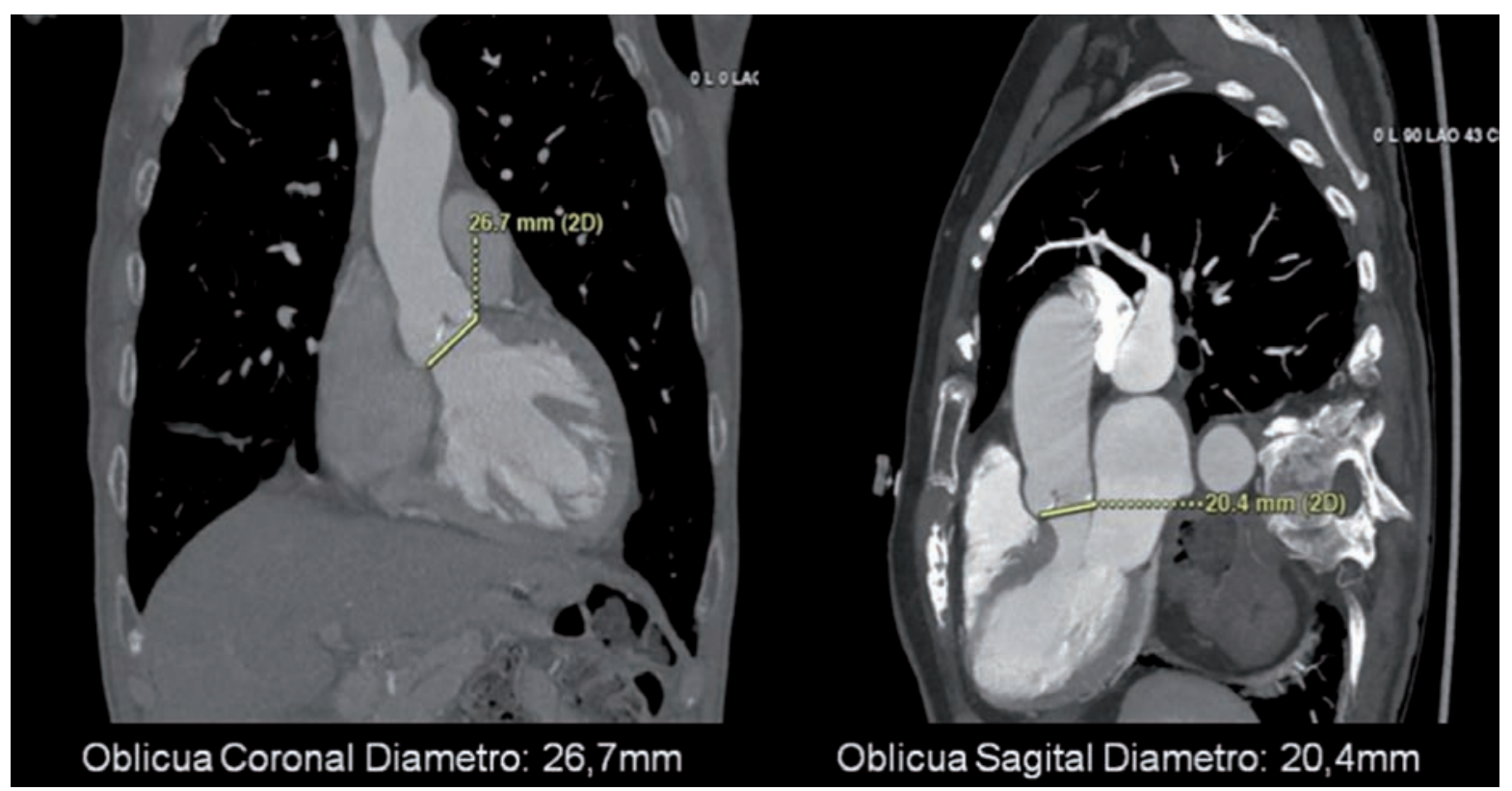

Figura 3. Reconstrucciones de angio-TC en proyecciones oblicuas para la medición del anillo.

la será la apropiada de acuerdo con las mediciones de perímetro y/o área del anillo. La selección de la prótesis basada en la medida del perímetro y/o área del anillo parece tener menor incidencia de leaks periprotésicos que cualquier otra medición. En casos donde no se disponga de angio-TC o se desee complementar con otro estudio, la ecografía transesofágica con reconstrucción 3D parece ser también un buen método de interpretación y medición de dicha estructura ${ }^{18}$.

\section{Recomendaciones}

\section{Clase I}

Se debe realizar angio-TC con contraste gatillada con cortes finos en todos los pacientes que puedan ser evaluados para TAVI, a fin de realizar las medidas del anillo aórtico y sus estructuras adyacentes para la elección del tipo y tamaño de prótesis (Nivel de Evidencia: C).

\section{Clase IIa}

Se puede utilizar el ecocardiograma transesofágico como complemento o alternativa de la angio-TC en casos donde la válvula aórtica no esté severamente calcificada (Nivel de Evidencia: C).

\section{Clase III}

La ecografía 2D (transesofágica o transtorácica) y la angiografía no son suficientes para realizar medidas adecuadas del anillo aórtico para planificar un TAVI (Nivel de Evidencia: C).

El anillo aórtico es una estructura muy importante para la selección correcta de la prótesis percutánea, pero no la única. La angulación del anillo respecto al ventrículo izquierdo (VI), el diámetro de los senos de Valsalva, la unión sinotubular, la altura de las coronarias y la aorta ascendente son importantes ${ }^{19}$. La angulación del anillo respecto al VI se mide en la angio-TC en la proyección oblicua coronal trazando una línea paralela al ani- llo valvular aórtico y otra línea paralela al diafragma. La unión de estas dos rectas genera el ángulo de implante. Los ángulos mayores a 30 grados demandarán mayor dificultad para el implante por la vía femoral o subclavia izquierda, desaconsejándose la vía subclavia derecha. Por vía transaórtica cualquier ángulo es aceptable, incluso ángulos de 90 grados (Figura 4A). El diámetro del seno de Valsalva y la altura del nacimiento de las coronarias son de capital importancia. Si bien la oclusión de ostia coronarios durante los implantes es una complicación infrecuente, cuando ocurre es de difícil resolución y alta mortalidad ${ }^{20}$. Para medir correctamente esta estructura se debe llevar el plano del anillo hasta el seno de Valsalva y en el corte doble oblicua axial realizar las mediciones de los tres senos (Figura 4B). Esta medición se efectúa rotando el eje del cursor $3 \mathrm{D}$ en la proyección doble oblicua axial hasta ubicar en la oblicua coronal el nacimiento de ambas coronarias. Una vez ubicado el nacimiento de la arteria se traza una línea de medición desde el plano del anillo hasta el punto inferior del nacimiento de la misma (Figura 4C). Dependiendo del tipo de prótesis, existen valores mínimos considerados de "seguridad" para evitar esta complicación. El diámetro de la aorta ascendente también es relevante. Se realiza trazando una línea paralela al anillo a $40 \mathrm{~mm}$ del mismo. En algunas prótesis autoexpandibles se requiere un diámetro mayor a fin de que pueda apoyarse la parte superior de la válvula. Eso no suele suceder con las válvulas balón expandibles.

La angiografía, si bien podría ser orientativa, no sería un método de primera opción para evaluación del tipo y tamaño de la prótesis ni de la elección del acceso. Sin embargo, la cinecoronariografía es un estudio útil y necesario para evaluar la presencia de enfermedad coronaria concomitante. La gran cantidad de calcio en la válvula aórtica y arterias coronarias ha- 


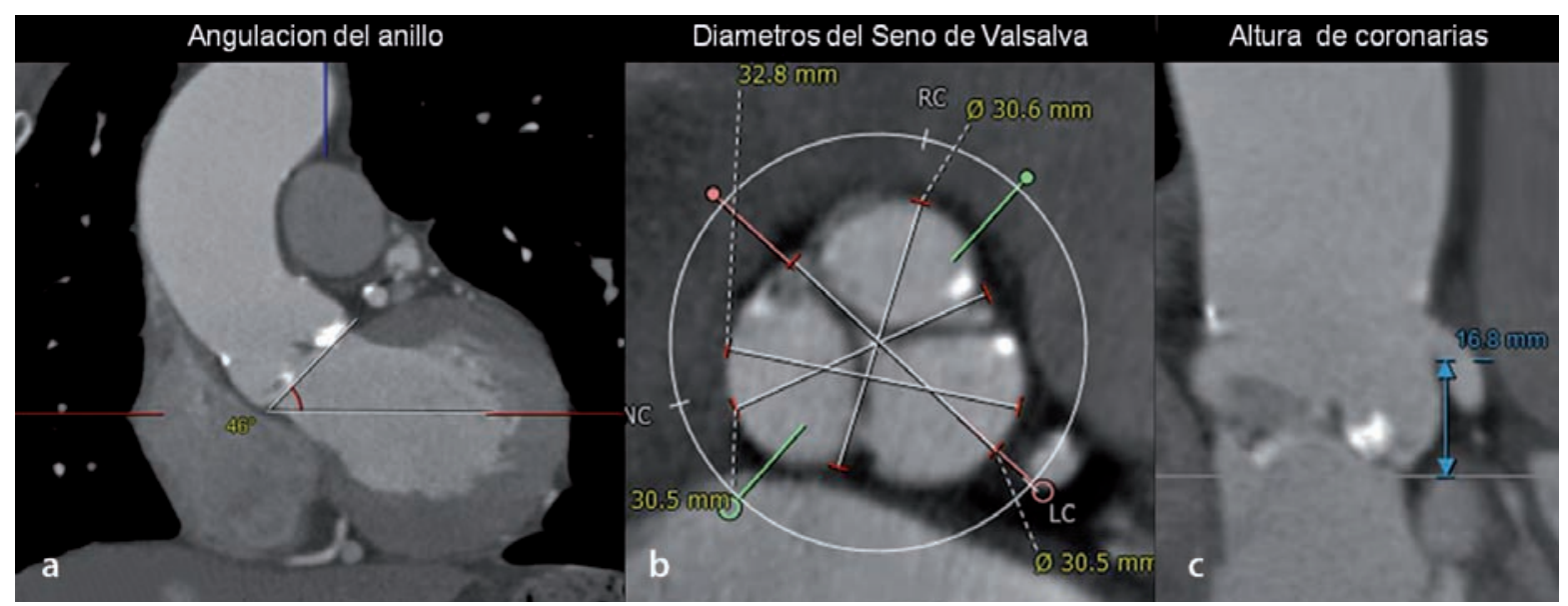

Figura 4. A: Medición de la angulación del anillo. B: Medición de los diámetros de los senos del Valsalva. C: Medición de la altura del ostium del tronco de la coronaria izquierda.

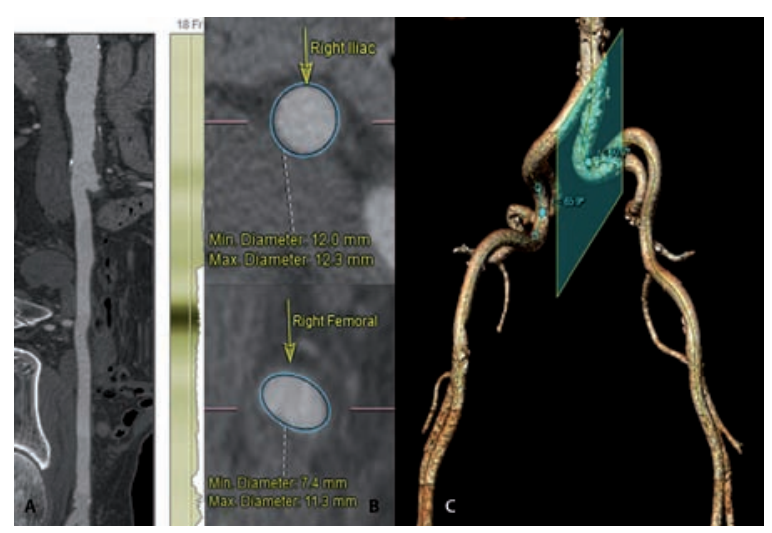

Figura 5. Medición de los accesos aorto-ilíaco-femorales por angio-TC. A: Reconstrucción longitudinal derecha. B: Mediciones de los diámetros en el corte axial. C: Reconstrucción 3D.

cen que la evaluación coronaria mediante angio-TC pueda no ser precisa al evaluar el grado de estenosis de esos vasos.

\section{Recomendaciones}

\section{Clase I}

Se deberá realizar una cinecoronariografía diagnóstica en todos los pacientes que sean candidatos a tratamiento mediante TAVI (Nivel de Evidencia: C).

\section{Clase IIa}

Se utilizará el acceso radial para la cinecoronariografía, a fin de preservar los accesos femorales para el eventual implante de TAVI (Nivel de Evidencia: C).

\section{Clase IIb}

La angio-TC coronaria multicorte puede ser una alternativa diagnóstica en pacientes con bajo score de calcio coronario $(<400)$ donde la calidad de las imágenes permita evaluar la anatomía coronaria en su totalidad (Nivel de Evidencia: C).

\subsection{Estudio de accesos vasculares}

La angio-TC es una gran herramienta para la evaluación de accesos periféricos para el TAVI. La reconstrucción 3D de los vasos aorto-ilíaco-femorales ofrece información sobre

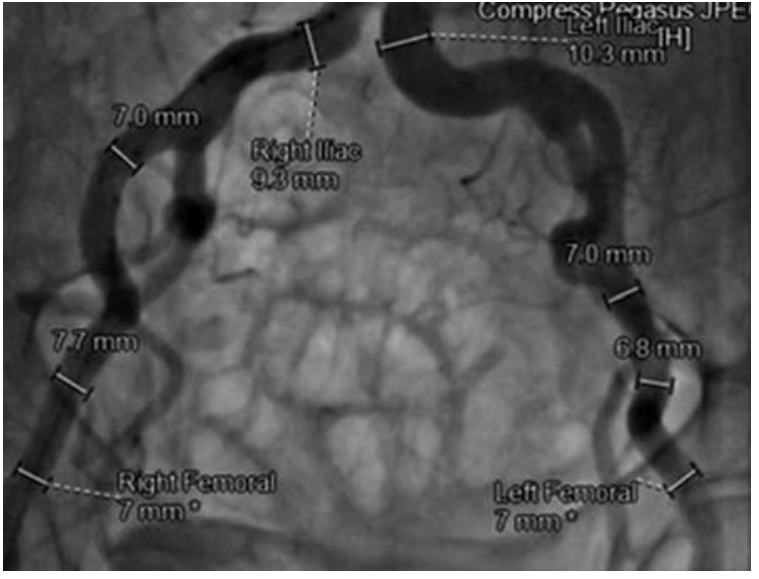

Figura 6. Medición de los accesos aorto-ilíaco-femorales por angiografía.

la tortuosidad y calcificación. La evaluación en la vista estirada (streched view) permite evaluar en cualquier punto el diámetro de la arteria en su totalidad (Figura 5). El acceso por arteria subclavia también puede ser evaluado por angio-TC. La angiografía sin técnica de sustracción con bomba infusora de contraste (30 cc al menos) por encima de la bifurcación ilíaca hasta la bifurcación de ambas femorales con pig tail centimetrado es una válida alternativa ya que permite evaluar los diámetros ilíacos y femorales, la altura de la bifurcación femoral (para eventual uso de dispositivos de cierre percutáneo), la distribución y cantidad de calcificación y la presencia de tortuosidades (Figura 6).

La evaluación de las arterias subclavias se puede efectuar con angiografía selectiva con pig tail centimetrado (Figura 7). La angiografía de aorta ascendente es útil para evaluar la posibilidad de acceso aórtico directo así como también el sitio de acceso, el cual se debe realizar a $60 \mathrm{~mm}$ del plano del anillo (Figura 8).

\section{Recomendaciones}

\section{Clase I}

La angio-TC con contraste representa el método de elección para evaluar la anatomía vascular periférica para la elección del sitio de acceso (Nivel de Evidencia: C). 


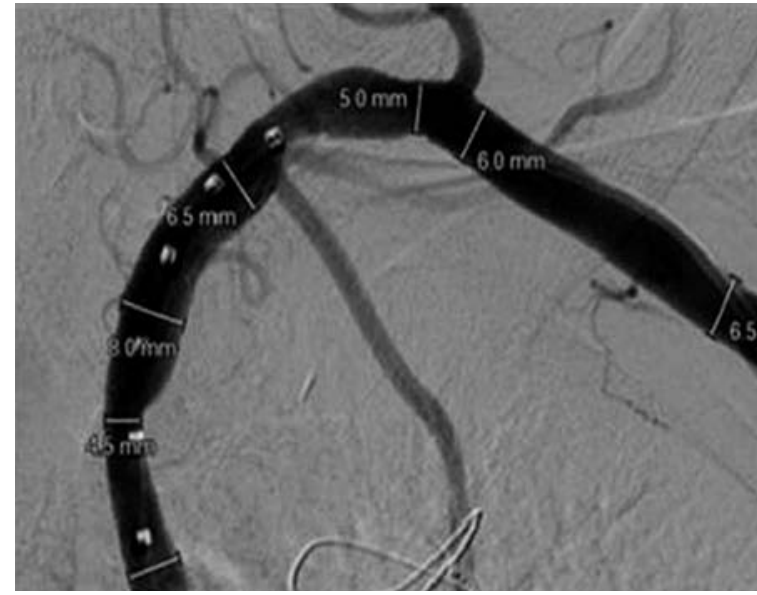

Figura 7. Medición del acceso subclavio izquierdo por angiografía.

\section{Clase IIb}

La angiografía digital con pig tail centimetrado representa una alternativa en los casos en los que no esté disponible la angio-TC (Nivel de Evidencia: C).

\section{ACCESOS}

La evaluación de las vías de acceso para el TAVI son un requisito fundamental a la hora de evaluar la factibilidad de la intervención, ya que si bien los dispositivos disponibles han ido disminuyendo su tamaño con respecto a los de primera generación todavía se requiere que la vía de acceso elegida tenga un diámetro no menor de $6 \mathrm{~mm}$ de diámetro. La mayoría de las bioprótesis tienen vainas que van de 16 a 22 french.

Existen dos grupos de accesos de acuerdo a su disposición respecto al anillo aórtico: uno anterógrado, conformado únicamente por la vía transapical, y otro retrógrado, conformado por la vía femoral (la más utilizada y recomendada), la vía subclavia y la vía transaórtica directa. También existen reportes de esta vía a través del acceso carotídeo y el acceso transcava (que luego se une al acceso aórtico retrógrado). La vía transfemoral es la más utilizada en nuestro país y en el mundo, pero existe un porcentaje de pacientes con enfermedad vascular periférica que no son aptos para ninguna de las vías retrógradas, lo que derivó en el desarrollo de la vía transapical.

\subsection{Tipos de acceso}

\section{Acceso transfemoral}

Esta vía permite realizar el abordaje de la arteria femoral mediante dos técnicas, por disección a cielo abierto que se realiza con una incisión a nivel del pliegue inguinal y que permite acceder a la cámara femoral a la cual accedemos por punción con aguja para colocar posteriormente el introductor a utilizar para el ascenso de la prótesis. Esta técnica es segura y permite tener visión directa de la arteria y control sobre la misma para su posterior cierre y control de hemostasia. Sin embargo, no está exenta de complicaciones siendo las más frecuentes las infec-

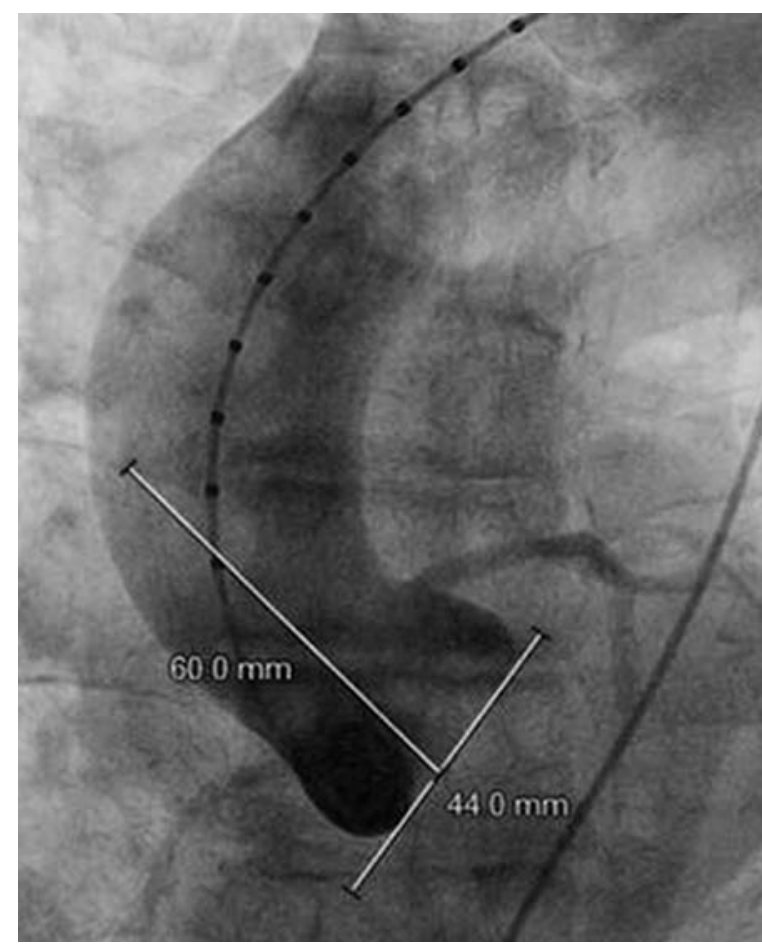

Figura 8. Medición del acceso transaórtico y aorta ascendente por angiografía.

ciones posoperatorias y los seromas, que aumentan la estadía hospitalaria y prolongan la deambulación. La alternativa es el acceso mediante punción de la arteria femoral y la utilización de dispositivos de sutura percutánea que permite realizar la intervención en forma percutánea. En este sentido resulta fundamental la selección de los pacientes pasibles de ser intervenidos de esta forma, descartando arterias calcificadas de tamaño menor a $6 \mathrm{~mm}$, y pliegues inguinales hostiles por intervenciones previas. La técnica consiste en realizar la punción sobre la pared anterior bajo control angiográfico (acceso femoral contralateral) a fin de asegurarse de que las agujas del dispositivo salgan en posición correcta para una adecuada hemostasia. Esta última técnica es menos invasiva pero requiere mayor entrenamiento por parte de los intervencionistas.

\section{Acceso transapical}

Esta vía de acceso es más invasiva que la transfemoral y está asociada a una tasa mayor de complicaciones. Se realiza bajo anestesia general mediante una toracotomía mínima antero lateral izquierda de $5 \mathrm{~cm}$ en el sexto espacio intercostal. Se expone el ápex, y se asegura la punta con suturas con pledget de teflón rodeando la zona a punzar. Se punza el ápex atravesando una cuerda 0,035 " que cruza por proximidad la válvula aórtica estenótica desde el ventrículo izquierdo a la aorta torácica, posteriormente se implanta la válvula y finalmente se retira el dispositivo y se procede al cierre de la jareta. Para los dispositivos que requieren marcapaseo a alta frecuencia durante la liberación de la prótesis, se pueden colocar cables de marcapaso epicárdico (Figura 9). 


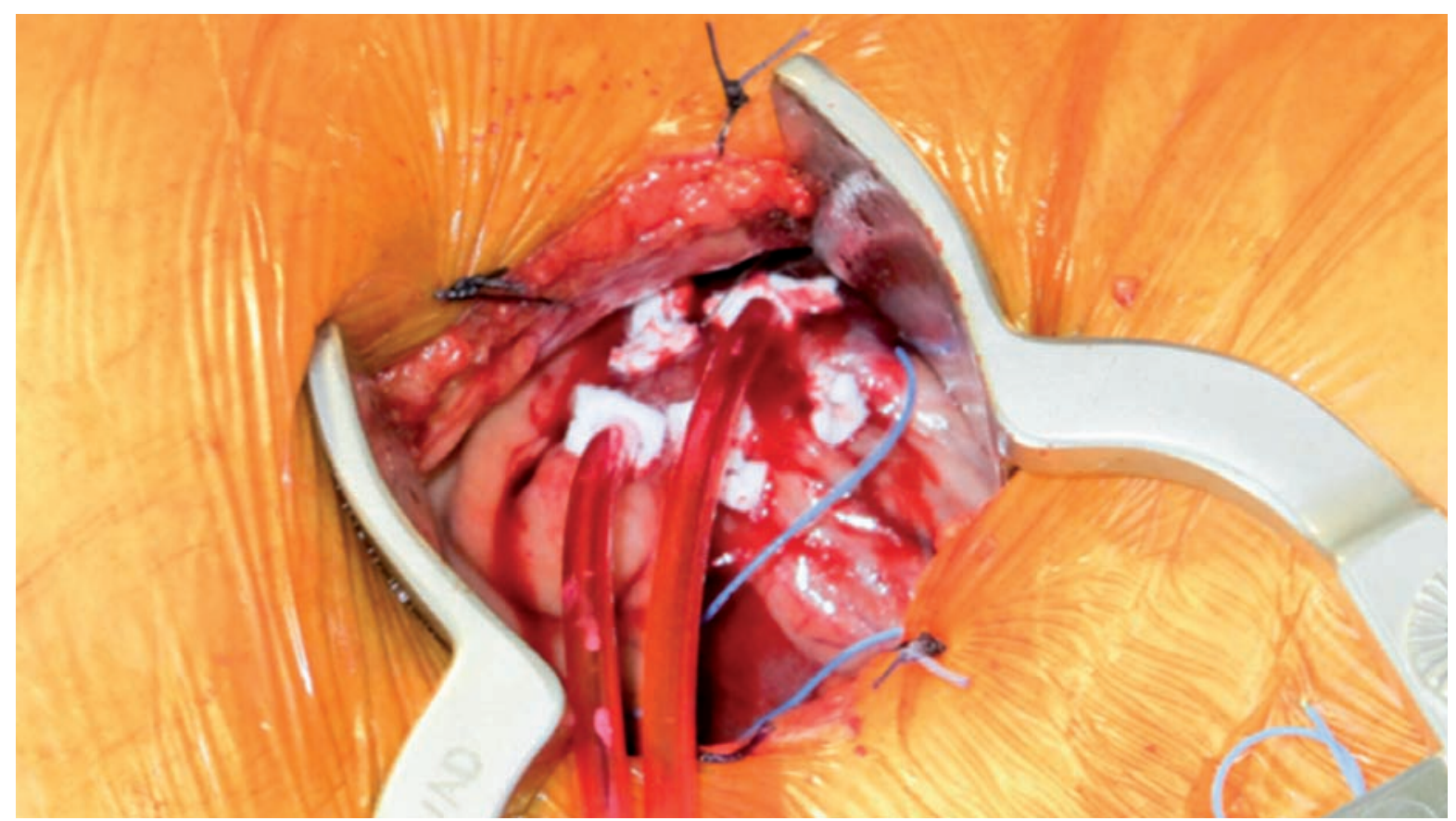

Figura 9. Abordaje transapical.

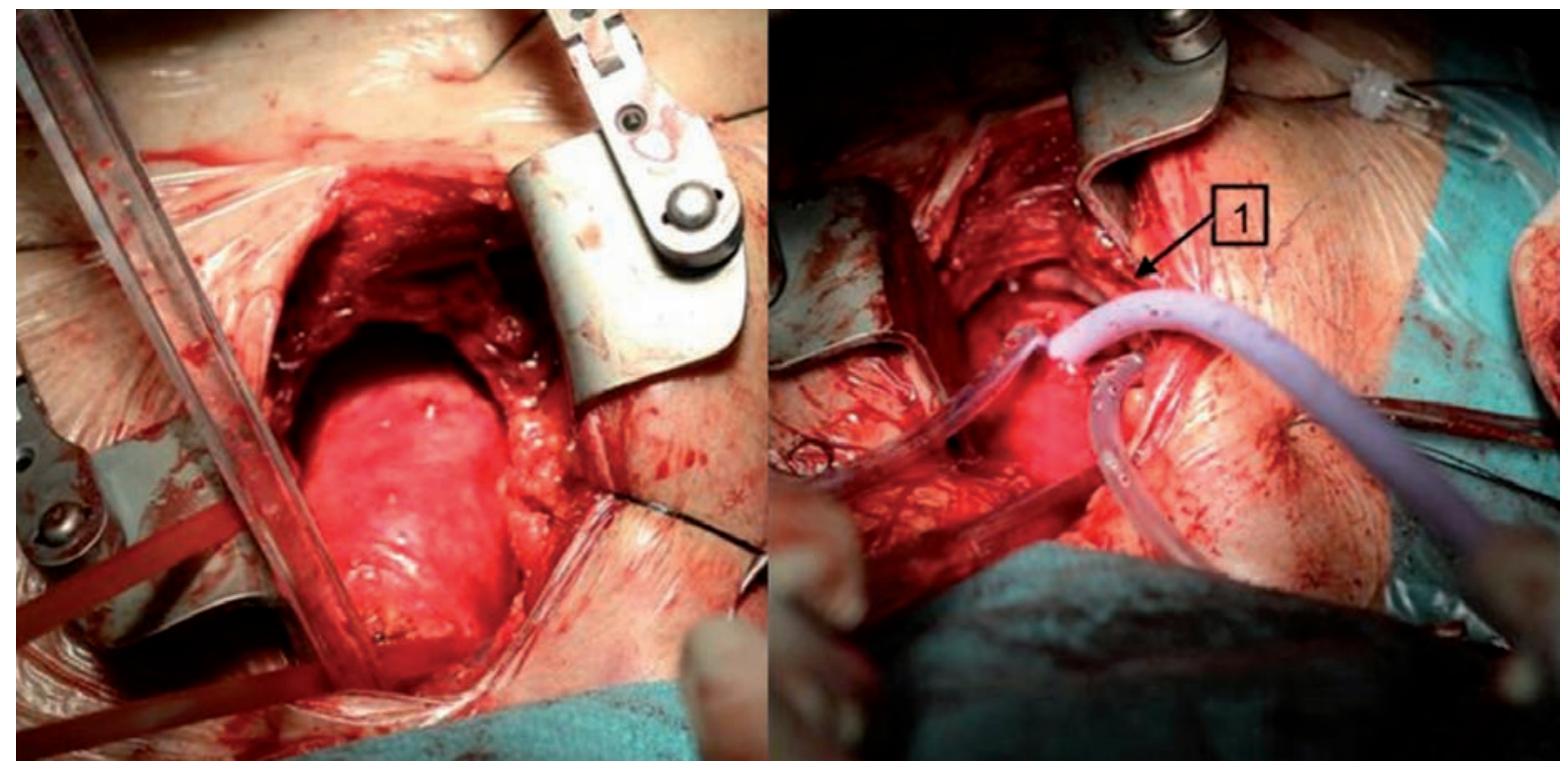

Figura 10. Abordaje transaórtico.

\section{Acceso transaórtico directo}

La técnica de implante transaórtica es una alternativa para pacientes cuyos accesos femoral y transubclavio no son viables, y consiste en una esternotomía mínima (Figura 10) desde el manubrio esternal hasta el borde esternal derecho a nivel del tercero o el cuarto espacio intercostal para la exposición de la aorta ascendente. Seguidamente se punza la pared anterior de la aorta y se coloca un introductor para permitir el ascenso del dispositivo a través de una cuerda soporte extra en el ápex del ventrículo izquierdo. Finalmente se retira el introductor y se realiza el cierre de la pared mediante una jareta que se hace al momento de la punción. Se debe dejar suficiente espacio desde el extremo distal del introductor a la prótesis para poder desplegarla adecuadamente, por lo que se aconseja un acceso lo más distal posible.

\subsection{Resultados de los accesos}

El estudio PARTNER-I ha demostrado que el acceso transapical se asociaba con mayor tasa de ictus y mortalidad precoz respecto del femoral. Sin embargo, estos resultados podrían deberse a las diferentes características basales de los pacientes de ambos grupos. Debido a la falta de trabajos aleatorizados para comparar ambos grupos, se realizó un score de propensión con el objetivo de eliminar factores confusionales y comparar ambas vías ${ }^{21}$. De esta forma, se analizaron 1521 pacientes por vía transfemoral (TAVI 
TF) y 1100 pacientes por vía transapical (TAVI TA). Se hallaron diferencias significativas de las características basales de ambos grupos, siendo más graves los pacientes del grupo TAVI TA. El score de propensión se realizó considerando 111 variables y el seguimiento mediano fue a un año. Este estudio mostró que los pacientes TAVI TA tuvieron mayor estadía hospitalaria (8vs. 5 días; $\mathrm{p} \leq 0,0001)$, menor recuperación de clase funcional en los primeros 30 días (31 vs. 38\% en NYHA I) y mayor número de eventos periprocedimiento. Se observó además una mayor mortalidad $(7,4$ vs. $2,8 \% ; \mathrm{p}=0.001)$ y recibieron con mayor frecuencia transfusiones de sangre $(4,2$ vs. $2 \%)$. Durante el procedimiento el tiempo medio de fluoroscopia $(22$ vs. 12 minutos) y la utilización de contraste $(120 \mathrm{ml}$ vs. $90 \mathrm{ml}$ ) fue superior en el grupo TAVI TF. También fue superior la regurgitación periprotésica en el grupo TAVI TF (57 vs. 35\%; $\mathrm{p}=0,001$ ) persistiendo esa diferencia a los 30 días y los 6 meses, aunque en la mayoría de los casos era de grado leve. De esta forma podemos concluir que, en pacientes con similar grado de arteriopatía periférica y factores de riesgo cardiovascular, el acceso transapical se asocia con mayor morbimortalidad que el femoral, y recomiendan este último como vía de elección principal para el TAVI.

\section{Recomendaciones}

\section{Clase I}

Se recomienda el acceso transfemoral como vía de acceso de elección. En caso de no ser viable esta vía, se recomienda utilizar cualquier otra vía retrógrada (transubclavia, transaórtica directa) o la vía transapical ( $\mathrm{Ni}$ vel de Evidencia: B).

\section{Clase IIb}

En caso de inviabilidad de la vía transfemoral, podrá optarse por la vía transapical en lugar de otras vías retrógradas en centros con experiencia en esta vía donde la tasa de complicaciones de la vía transapical sea menor que la de las otras vías retrógradas (Nivel de Evidencia: C).

\section{COMPLICACIONES}

Con el objetivo de mejorar la calidad de los ensayos clínicos y poder efectuar comparaciones entre ellos, en el año 2011 se realizó un consenso internacional con definiciones de puntos finales clínicos de eficacia y seguridad $^{22}$. Dicho consenso denominado VARC (Vat ve Academic Research Consortium) detalla, entre otras cosas, las siguientes complicaciones y fue actualizado recientemente ${ }^{23}$.

\subsection{Leak paravalvular}

El leak o fuga paravalvular es una complicación frecuente del TAVI y su hallazgo es más frecuente que en los pacientes sometidos a reemplazo valvular aórtico quirúrgico. Dependiendo del método de evaluación (angiografía, ecocardiografía, mediciones cuantitativas o semicuantitativas) la prevalencia reportada de leak paravalvular varía entre 40 y $67 \%$ para leaks triviales y leves, y entre 7 y $20 \%$ para leaks moderados a severos ${ }^{24}$. Existe evidencia de la asociación entre la presencia de leak paravalvular moderado a severo con la mortalidad a corto y largo plazo post-TAVI ${ }^{10}$. En un metaanálisis de 45 estudios incluyendo 12.926 pacientes (CoreValve $\mathrm{n}=5261$ y Edwards $\mathrm{n}=7279$ ) se observó una incidencia de leak moderado a severo del $11,7 \%{ }^{24}$. La presencia de leak moderado a severo se acompañó de mayor mortalidad a 30 días. En este estudio, la profundidad del implante, la subvaloración de la prótesis y el score de calcio de Agatston fueron identificados como predictores importantes de leak paravalvular ${ }^{24}$. El desarrollo de nuevas generaciones de prótesis, reposicionables y con diseños que incluyen mecanismos de mejor adaptación y aposición a la anatomía del anillo valvular y estructuras adyacentes ha logrado disminuir la incidencia de leak en relación con las primeras generaciones. Un registro prospectivo $(n=60)$ de tratamiento con el sistema CoreValve Evolut R reportó una incidencia de leak paravalvular moderado de 3,4\% sin observarse leak severo ${ }^{25}$, mientras que se observó un 3,4\% de leak moderado a severo observado con la válvula balón expandible SAPIEN $3^{26}$ y un $1 \%$ de leak moderado sin leak severo con la válvula Lotus ${ }^{27}$. Dado que no existe un único método de diagnóstico que permita evaluar con exactitud el mecanismo y la severidad de un leak paravalvular posimplante, se sugiere utilizar la combinación de varios métodos junto con la evaluación hemodinámica. El índice de regurgitación aórtica $(\text { IRA })^{28}$, calculado como la diferencia entre la presión diastólica aórtica (PDAo) y la presión de fin de diástole del ventrículo izquierdo (PFDVI) dividida la presión sistólica aórtica (PSAo)

IRA $=($ PDAo - PFDVI $) /$ PSAo $\times 100$,

ofrece una evaluación fisiológica inmediata y proporciona información pronóstica. Los pacientes con IRA $<25$ presentaron un significativo aumento de la mortalidad al año en comparación con aquellos con IRA $\geq 25$ ( 46 vs. 16,7\%; $<<0,001$ ). La resonancia magnética cardíaca podría ser utilizada en la evaluación alejada post-TAVI de la magnitud de la regurgitación paravalvular.

\section{Tratamiento del leak paravalvular post TAVI:}

1. Prevenir es mejor que tratar. La correcta medición del anillo valvular y las estructuras adyacentes son fundamentales para la elección del tipo y tamaño de la prótesis, reduciendo la incidencia de leak paravalvular.

2. Tratamiento (depende de la severidad y el mecanismo). Se proponen los siguientes tratamientos. Estos se enumeran en relación con su complejidad (de menor a mayor), por lo que se sugiere seguir el orden establecido: 
a. Reevaluar luego de un tiempo prudencial (aproximadamente 10 minutos). Esto puede ser de particular ayuda en las válvulas autoexpandibles.

b. Posdilatación con balón.

c. Colocación de una segunda válvula (valve-in-val$v e$ ). Este tratamiento se utiliza sobre todo en casos de inapropiada altura del implante.

d. Cierre percutáneo del leak mediante plug vasculares.

e. Reemplazo valvular aórtico quirúrgico. Este tratamiento debe quedar reservado para situaciones excepcionales.

En casos muy seleccionados y cuando el leak se deba a una posición baja de la prótesis, el reposicionamiento mediante tracción con un lazo puede ser una alternativa.

\subsection{Bloqueo auriculoventricular}

Las alteraciones de la conducción eléctrica constituyen una complicación relativamente frecuente durante el TAVI, y se deben a la proximidad anatómica entre el complejo valvular aórtico y el sistema de conducción eléctrica cardíaco. Los mecanismos de injuria al sistema de conducción incluyen la compresión directa, hemorragia, hematoma, injuria isquémica e inflamación. El bloqueo de rama izquierda (BRI) es el trastorno más frecuente $83 \%$, seguido por el bloqueo A-V completo (19\%) y el bloqueo de rama derecha (BRD, 8\%). En algunos estudios se observó hasta un $50 \%$ de nuevo trastorno de conducción durante la valvuloplastia aórtica (46\%) y la manipulación de cuerdas de alto soporte en ventrículo izquierdo $(4 \%)^{29}$.

En un metaanálisis reciente $(\mathrm{n}=11.210)$ se observó una tasa de implante de marcapaso (MCP) definitivo del $17 \%$, identificándose al género masculino, alteraciones basales de la conducción eléctrica (bloqueo $\mathrm{A}-\mathrm{V}$ de primer grado, hemibloqueo anterior, bloqueo de rama derecha) y el desarrollo del bloqueo AV completo intraprocedimiento, como predictores de implante de MCP definitivo. La necesidad de MCP definitivo fue 2,5 veces mayor con la prótesis autoexpandible que con la balón expandible ${ }^{30}$. Otro de los factores asociados a la necesidad de marcapasos es la profundidad del implante ${ }^{10}$.

De manera similar a lo que ocurre luego del reemplazo valvular aórtico quirúrgico, los trastornos de conducción pueden ser reversibles, por lo que se requiere, en algunos casos, un período de observación antes de la colocación de un MCP definitivo ${ }^{31}$. Sin embargo, la duración de este período de observación no está definida. Algunos estudios con seguimiento a largo plazo han mostrado ausencia de impacto de la necesidad de MCP definitivo sobre mortalidad, aunque esta complicación se asocia a prolongación de la hospitalización y empeoramiento de insuficiencia cardíaca en el seguimiento ${ }^{32}$.

La meticulosidad en la técnica de implante de las prótesis aórticas (implante más alto) y las modificaciones en los nuevos diseños son fundamentales para disminuir el trauma sobre el sistema de conducción eléctrica y minimizar la necesidad de MCP definitivo, sobre todo cuando se pretende expandir las indicaciones de TAVI a poblaciones de mediano o bajo riesgo.

\section{Recomendaciones}

\section{Clase I}

Se recomienda al monitoreo continuo hasta 72 horas luego del implante para la detección de arritmias ${ }^{23}(\mathrm{Ni}-$ vel de Evidencia: C).

\subsection{Complicaciones del acceso}

Las complicaciones del acceso vascular son causa mayor de morbilidad y mortalidad, y han sido descriptas con una frecuencia del $12-30 \%$ en distintos estudios ${ }^{33}$. El consenso VARC-2 incluye a todas las complicaciones ocasionadas por una cuerda guía, un catéter, o cualquier otro elemento relacionado con el acceso, incluyendo perforación ventricular izquierda o pseudoaneurisma ventricular en caso de acceso transapical ${ }^{23}$. Se define como complicaciones mayores aquellas que resultan en mortalidad, sangrado mayor, isquemia visceral, embolización vascular distal, que requiere intervención quirúrgica o amputación. Dentro de las complicaciones más frecuentes, se han descripto: disección, estenosis, oclusión, perforación, ruptura, fístula arteriovenosa, pseudoaneurisma, hematoma, injuria irreversible de nervio periférico, síndrome compartimental o fracaso del dispositivo de cierre percutáneo. Diferentes estudios han evaluado los predictores de complicaciones del acceso transfemoral. Dentro de los predictores de complicaciones del acceso podemos identificar al sexo femenino, y a la utilización de introductores mayores de 19 french $^{34}$. Independientemente del tamaño del dispositivo, la relación entre el diámetro externo de la vaina de acceso vascular y el diámetro arterial (SFAR) $\geq 1,05$ es también un predictor independiente de complicaciones vasculares mayores $(30,9$ vs. $6,9 \% ; \mathrm{p}=0,001)$ y de mortalidad a 30 días $(18,2$ vs. $4,2 \% ; \mathrm{p}=0,016)^{35}$.

El tratamiento tradicional de estas complicaciones es la reparación quirúrgica. Sin embargo, el tratamiento percutáneo mediante insuflaciones prolongadas y colocación de stents forrados es efectivo para resolver la mayoría de las disecciones, estenosis, obstrucciones, perforaciones o sangrado secundario a cierre incompleto del acceso vascular ${ }^{25,36}$.

Existen, además, complicaciones específicas para los accesos transapical, axilo-subclavio y transaórtico ${ }^{37}$.

\section{Acceso transapical:}

- Sangrado de punción apical o desgarro miocárdico que requiere reparación quirúrgica inmediata.

- Fibrosis apical, que determina hipoquinesia segmentaria con impacto en FSVI. global, especialmente relevante en pacientes con deterioro previo de FSVI.

- Formación de aneurismas.

- Lesión de nervios intercostales. 


\section{Acceso subclavio:}

- Injuria vascular, con disección arterial, oclusión trombótica. (solucionables de forma endovascular o quirúrgica).

\section{Acceso transaórtico:}

- Desgarro de la aorta que requiere reparación quirúrgica.

- Infección de la herida.

- Mediastinitis.

- Lesión de arteria mamaria interna derecha (minitoracotomía anterior derecha)

Los avances como la disminución del perfil de los introductores, la evaluación del acceso mediante imágenes, la selección del acceso vascular más apropiado y la creciente experiencia de los operadores, contribuyen a disminuir la incidenciaes estas complicaciones

\subsection{Stroke}

El stroke constituye una complicación de baja incidencia $(2,5-3 \%)^{38}$ pero alta morbimortalidad cuando se trata de un stroke mayor. De acuerdo con los criterios VARC-2, se define stroke como un episodio agudo de disfunción neurológica focal o global, causada por injuria vascular cerebral, medular o retiniana. El stroke puede ser isquémico o hemorrágico. Un stroke puede clasificarse como "indeterminado" si no hay información suficiente para permitir categorizarlo en isquémico o hemorrágico. El accidente isquémico transitorio (AIT) es una entidad estrechamente relacionada al stroke isquémico, pero se diferencia por acompañarse de déficit neurológico focal o global con duración menor de 24 horas y ausencia de alteración sugestiva de stroke agudo en neuroimágenes. De acuerdo con el consenso VARC- ${ }^{23}$, se recomienda utilizar la escala modificada de Rankin para diferenciar entre stroke incapacitante o no incapacitante. Se recomienda que un neurólogo vascular sea el encargado de evaluar los pacientes con stroke y definir la conducta (tratamiento farmacológico y/o invasivo). Algunos de los predictores de stroke son el sexo femenino, la enfermedad renal crónica, los procedimientos realizados durante la fase inicial de la experiencia del centro, y la fibrilación auricular de reciente aparición ${ }^{39}$. La posdilatación con balón mostró una tendencia a mayor riesgo de stroke aunque esta diferencia no fue estadísticamente significativa, al igual que no se encontraron diferencias según el tipo de válvula o el acceso. Tampoco se observaron diferencias según el anticoagulante (heparina o bivalirudina) utilizado durante el TAVI ${ }^{40}$. Existen sistemas de protección cerebral para la prevención del stroke durante el TAVI, aunque los resultados de los estudios iniciales han sido contradictorios ${ }^{41,42}$ y solo han demostrado reducir la incidencia de infartos lacunares asintomáticos medidos con resonancia magnética. En la actualidad ninguno de estos sistemas de protección cerebral se encuentra disponible en nuestro país.

\section{CUIDADOS POSIMPLANTE}

$\mathrm{Al}$ igual que otras intervenciones percutáneas, el TAVI tiene la posibilidad de generar eventos cardiovasculares adversos. Además, la implantación de un dispositivo sin la remoción de la válvula nativa genera zonas irregulares que alteran el flujo sanguíneo provocando estasis. Por otra parte, la exposición de los componentes metálicos del stent a los tejidos favorece la formación de material trombótico, en especial en válvulas de pequeño tama$\tilde{n} \mathrm{O}^{43}$. Es por ello que se requieren estrategias antitrombóticas y anticoagulantes durante y luego del TAVI.

\subsection{Terapia antitrombótica durante el TAVI}

La terapia antitrombótica óptima todavía está sujeta a debate debido a que existen pocos ensayos clínicos. Durante el implante de la válvula, desde los ensayos PARTNER trial ${ }^{11,13}$ la terapia más difundida es el uso de heparina no fraccionada (HNF) de un bolo de $5000 \mathrm{U}$ y luego bolos adicionales para mantener el tiempo de coagulación activado (ACT) $\geq 250$ segundos. Aunque un reciente consenso sugiere mantener el ACT en mayor a 300 segundos[6]. El uso de la protamina para revertir su exceso es recomendado a finalizar el implante. Se ha propuesto a la bivalirudina como anticoagulante $\mathrm{du}-$ rante el procedimiento, aunque a pesar de su vida media corta no puede ser revertida, y sus costos son mucho mayores. El estudio BRAVO 3 ( $\mathrm{n}=802)$ que comparó ambos antitrombóticos no encontró superioridad de la bivalirudina por sobre la heparina en relación a sangrados, aunque sí se alcanzó la no inferioridad ${ }^{44}$.

\subsection{Antiagregación luego del implante de TAVI}

A los pacientes que se les implanta un TAVI, la estrategia de antiagregación habitual es el uso de la doble antiagregación plaquetaria (DAPT), con aspirina y clopidogrel. En los ensayos PARTNER ${ }^{11,13}$ se utilizó aspirina (AAS) de 75 a $100 \mathrm{mg}$ por día en forma indefinida y clopidogrel $75 \mathrm{mg}$ por 6 meses con una dosis de carga de $300 \mathrm{mg}$ en los pacientes que no lo venían tomando. Los estudios que compararon la DAPT contra el tratamiento con aspirina sola son escasos, y no demostraron diferencias significativas en cuanto a puntos finales de trombosis ni de sangrado ${ }^{45-48}$.

En la Tabla 2 se observan las recomendaciones de terapia antiplaquetaria de algunas sociedades ${ }^{4,6,7}$.

\subsection{Anticoagulación}

Como fue explicado anteriormente, el TAVI podría ser protrombótico independientemente del estado de activación de las plaquetas. Además, luego de un reemplazo valvular aórtico quirúrgico con una prótesis biológica, algunos consensos recomiendan la anticoagulación en los primeros tres meses ${ }^{49}$. Recientemente se ha demostrado la trombosis in situ en la prótesis luego del TAVI, manifestado por elevación de los gradientes posprocedimiento, sin ser observados por el ecocardiograma transtorácico ni transesofági- 
TABLA 2. Terapia antitrombótica según las principales guías en pacientes con TAVI.

\begin{tabular}{|c|c|c|c|c|}
\hline & ESC guidelines ${ }^{4}$ & AHA/ACC guidelines ${ }^{5}$ & $\begin{array}{l}\text { ACCF/AAT/SCAI/STS } \\
\text { Consensus }^{6}\end{array}$ & $\begin{array}{l}\text { Canadian Society } \\
\text { Statement }^{7}\end{array}$ \\
\hline Aspirina & Dosis bajas indefinidamente & $\begin{array}{l}\text { Dosis bajas (75-100 mg) in- } \\
\text { definidamente }\end{array}$ & $81 \mathrm{mg}$ indefinidamente & Dosis bajas indefinidamente \\
\hline $\begin{array}{l}\text { Terapia antiplaquetaria } \\
\text { adicional }\end{array}$ & $\begin{array}{l}\text { Tienopiridina inmediatamente des- } \\
\text { pués del TAVI }\end{array}$ & $\begin{array}{l}\text { Clopidogrel } 75 \mathrm{mg} / \mathrm{dí} \text { por } \\
6 \text { meses }\end{array}$ & $\begin{array}{l}\text { Clopidogrel } 75 \mathrm{mg} / \mathrm{dí} \text { por } \\
\text { 3-6 meses }\end{array}$ & Clopidogrel por 3 meses \\
\hline Anticoagulación oral & $\begin{array}{l}\text { VKA solamente en pacientes con FA } \\
\text { y sin EC } \\
\text { VKA + terapia antiplaquetaria si hay } \\
\text { FA y hay un implantación de stent } \\
\text { reciente como en las guías de EC. }\end{array}$ & & $\begin{array}{l}\text { Si está indicado VKA (clopi- } \\
\text { dogrel no) }\end{array}$ & $\begin{array}{l}\text { Si está indicado VKA (evitar la } \\
\text { triple terapia, salvo que ten- } \\
\text { ga una indicación definitiva) }\end{array}$ \\
\hline
\end{tabular}

VKA: anticoagulantes Vit K dependientes (warfarina y acenocumarol). EC: enfermedad coronaria.

$\mathrm{co}^{50}$. Esto se observa con mayor frecuencia en válvulas grandes. En un caso incluso el tratamiento anticoagulante revirtió esta elevación de gradientes. A diferencia de la ecografía, la tomografía puede detectar estas trombosis al visualizar motilidad anormal de alguna de las valvas. Estudios recientes con angio-TC sugieren que las alteraciones de la motilidad de alguna de las valvas por trombosis tendrían una prevalencia que varía del $7 \%$ hasta el $40 \%^{51,52}$. Al igual que el caso mencionado anteriormente, en todos los pacientes que fueron tratados con anticoagulación este trastorno revirtiós $^{\text {1. }}$. El uso de los nuevos anticoagulantes orales luego del TAVI está siendo investigado en dos ensayos aleatorizados: el ATLANTIS (Anti-Thrombotic Strategy After Trans-Aortic Valve Implantation for Aortic Stenosis, NCT02664649) y el GALILEO (Global Study Comparing a Rivaroxaban-BasedAntithrombotic Strategy to an Antiplatelet-Based Strategy After Transcatheter Aortic Valve Replacement to Optimize Clinical Outcomes, NCT02556203) $)^{53}$.

\section{Recomendaciones}

\section{Clase I}

Durante la intervención se recomienda el uso de HNF a dosis necesarias para tener un $\mathrm{ACT} \geq 300$ segundos (Nivel de Evidencia: B).

En pacientes alérgicos a la HNF se recomienda el uso de bivalirudina en dosis anticoagulantes (Nivel de Evidencia: B).

Luego del TAVI se deberá indicar aspirina de $100 \mathrm{mg} /$ día en forma indefinida, y clopidogrel $75 \mathrm{mg}$ (dosis de carga de $300 \mathrm{mg}$ en aquellos pacientes que no venían tomando) por un período de 3 a 6 meses de acuerdo a eventos de sangrado (Nivel de Evidencia: C).

\section{Clase IIa}

Se sugiere en pacientes con incremento precoz de los gradientes una tomografía para detectar movimiento anormal de alguna de las hojas de la válvula. En caso de ser detectado, se deberá indicar la anticoagulación con antagonistas de la vitamina $\mathrm{K}$ por al menos tres meses (Nivel de Evidencia: B).

\subsection{Fibrilación auricular}

La fibrilación auricular es la arritmia más frecuente en la población general, con alta prevalencia en pacientes añosos, así como en pacientes con estenosis aórtica severa. LA FA y la estenosis aórtica coexisten en cerca del 30\% de los pacientes, con una prevalencia que varía entre el 16 y el $40 \%$ según las series ${ }^{54}$. El TAVI per se puede ocasionar el desarrollo de FA de nuevo inicio con una prevalencia que va del $0,7 \mathrm{al} 31 \%^{55}$. En la población general, independientemente del TAVI, se estima que la FA incrementa el riesgo de mortalidad en el hombre 1,5 veces y en la mujer 1,9 veces $^{56}$. Además, la FA luego del reemplazo de válvula aórtica, o posterior a una cirugía de revascularización miocárdica, es un predictor independiente de eventos cardiovasculares mayores ${ }^{57}$. En un metaanálisis reciente en TAVI, se observó que la FA preexistente se asoció con un aumento de la mortalidad cardiovascular a largo plazo, pero sin aumento en el stroke. En cambio, la FA de reciente inicio no se asoció a aumento de la mortalidad pero sí a mayor riesgo de stroke $e^{58}$.

\subsection{Seguimiento}

Los pacientes post TAVI son pacientes frágiles y presentan diferentes comorbilidades, por lo que el manejo es multidisciplinario.

La durabilidad de la prótesis de la TAVI es comparable a la de una válvula biológica. A pesar de su reciente introducción, solo el 3,4\% presentó reestenosis a los cinco años. El gradiente se ha mantenido estable en diferentes series sin incremento de la insuficiencia aórtica, aunque son necesarios seguimientos a más largo plazo ${ }^{59}$. En el seguimiento de los pacientes hay detalles que deben ser analizados con cuidado, entre estos elementos están el laboratorio, electrocardiograma, holter y ecocardiograma. Además, el monitoreo de sus comorbilidades. ${ }^{60}$. En resumen, se realiza un primer control a los 7 días del implante con ECG y especial control en el sitio de acceso. Luego otro control al mes (ECG, laboratorio) y con un control de ecocardiograma, luego al 3er mes (control clínico y ECG), luego del 6to mes con un nuevo ecocardiograma y finalmente al año con un nuevo ecocardiograma y laboratorio ${ }^{60}$.

\section{SITUACIONES ESPECIALES}

El TAVI ha evolucionado de ser una nueva tecnología a un tratamiento establecido en los pacientes con estenosis aórtica severa sintomática inoperable y de alto riesgo quirúrgico. El desarrollo de dispositivos de segunda generación, sumado a la mayor experiencia de los operadores, ha permitido reducir las complicacio- 
nes durante el procedimiento. De esta manera, las indicaciones actuales podrían en un futuro no muy lejano ampliarse a poblaciones de menor riesgo quirúrgico y con anatomías más desafiantes como las indicaciones que se discuten a continuación.

\subsection{Válvula bicúspide}

La válvula aórtica bicúspide es la cardiopatía congénita más frecuente en la población general, siendo su prevalencia del $0,5-2 \%{ }^{61} \mathrm{y}$, en aproximadamente el $50 \%$ de los casos, coexiste con una dilatación de la raíz aórtica o de la aorta ascendente. A pesar de la experiencia creciente del TAVI, los pacientes con estenosis aórtica de válvula bicúspide han sido excluidos inicialmente de los estudios aleatorizados y han sido considerados una contraindicación relativa debido a las dificultades técnicas relacionadas con la anatomía que las caracteriza e incluyen:

a. dificultad en el posicionamiento y anclaje del dispositivo debido a la dilatación de la aorta ascendente y a la forma extremadamente elíptica del anillo, pudiendo generar dislocación de la prótesis y obstrucción coronaria.

b. aposición incompleta de la válvula debido a una asimetría de las valvas asociada a la presencia de una severa y frecuentemente excéntrica calcificación, requiriendo posdilatación y estando asociada con peores resultados hemodinámicos ya sea por un mayor gradiente transvalvular como por un mayor desarrollo de insuficiencia aórtica paravalvular.

c. aumento del riesgo de ruptura de la raíz aórtica y de la aorta ascendente durante la valvuloplastia, el implante o la posdilatación.

d. la infraexpansión y consecuentemente la ausencia de una forma circular podrían afectar la durabilidad a largo plazo.

A pesar de esto, existe una creciente evidencia en este contexto. Las series iniciales con dispositivos de primera generación han demostrado que el TAVI es un procedimiento factible en pacientes de alto riesgo quirúrgico con válvulas bicúspides, sin embargo se observó una mayor tasa de insuficiencia aórtica paravalvular comparado con las válvulas tricúspides ${ }^{62-64}$. El desarrollo de nuevos dispositivos con mejoras estructurales y de diseño demostraron resultados favorables con menor tasa de insuficiencia aórtica paravalvular que las primeras generaciones en pacientes con válvula aórtica bicúspide. El Registro TAVI en Válvula Bicúspide $(\mathrm{n}=301)$ mostró una menor tasa de insuficiencia aórtica paravalvular moderada o severa con las nuevas generaciones (SAPIEN 3 y Lotus) que con válvulas anteriores (SAPIEN XT y CoreValve) ( $0 \% v s .8,5 \%$; $\mathrm{p}=0.002)^{64}$. Eso se vio asociado a una mayor tasa de éxito del dispositivo $(92,2 \%$ vs. $80,9 \%$; $\mathrm{p}=0.01)$, sin diferencias en la tasa de $\mathrm{ACV}$ (2,5\% vs. 2,0\%), sangrado que compromete la vida ( $3,5 \%$ vs. $2,9 \%)$, complicaciones vasculares mayores ( $4,5 \%$ vs. $2,9 \%)$, ni en la mor- talidad a 30 días (4,5\% vs. 3,9\%). Si bien se necesitan estudios adicionales con un mayor número de pacientes para evaluar el resultado de las nuevas generaciones de válvulas en este tipo de pacientes, estas podrían ser una opción razonable para contrarrestar las dificultades técnicas asociadas a la anatomía de las válvulas bicúspides y podrían hacer del TAVI una alternativa en pacientes seleccionados.

\subsection{Insuficiencia aórtica pura}

El TAVI para el tratamiento de los pacientes con insuficiencia aórtica pura se ve limitado por la ausencia de calcificación anular o valvar, lo que podría no solo aumentar el riesgo de dislocación o embolización de la válvula secundario a un anclaje inadecuado, sino que llevaría a realizar un oversizing pudiendo favorecer la ruptura del anillo. Por esta razón, la cirugía de reemplazo valvular aórtico continúa siendo el tratamiento de elección para los pacientes que pueden someterse a este procedimiento. La presencia de una combinación de estenosis severa e insuficiencia aórtica no representa una contraindicación para TAVI, como así tampoco la presencia de insuficiencia aórtica severa en una válvula protésica (valve in valve). Los estudios iniciales utilizaron la válvula CoreValve dado que posee la posibilidad de anclaje en su porción superior a la aorta ascendente, contribuyendo a una mejor estabilización del dispositivo; y la JenaValve dado su mecanismo de anclaje a nivel de las cúspides nativas, similar a los clips metálicos para papeles. Se han descripto también TAVI con la válvula ACURATE TA y Direct Flow. Un reciente metaanálisis ( $\mathrm{n}=237$ en 13 estudios) mostró una tasa de éxito entre el $74 \%$ y el $100 \%$, pero que $7 \%$ de los pacientes requirieron de una segunda válvula y $2,5 \%$ de conversión a cirugía convencional ${ }^{65}$. El TAVI en pacientes con insuficiencia aórtica pura, por el momento debería estar limitado a los pacientes inoperables o con riesgo quirúrgico prohibitivo, dado que la evidencia clínica acumulada no es suficiente para establecer no inferioridad del TAVI comparado con la cirugía.

\subsection{Valve in valve}

En la actualidad, las válvulas biológicas se prefieren sobre las mecánicas debido a la reducida trombogenicidad, evitando de esta forma la necesidad de anticoagulación. Sin embargo, tienen una durabilidad limitada de alrededor de 10-20 años. El deterioro estructural de las prótesis quirúrgicas biológicas pueden manifestarse hemodinámicamente con estenosis, insuficiencia o la combinación de ambas. Algunos de los mecanismos involucrados son: calcificación, fractura, formación de pannus, trombosis valvular y endocarditis. El TAVI en esta población representa una alternativa cuando la recirugía es de riesgo prohibitivo o de alto riesgo quirúrgico. El Registro Valve in Valve ( $\mathrm{n}=202,61 \%$ CoreValve y 39\% SAPIEN) mostró un éxito del procedimiento del $93,1 \%$ y una mortalidad del $8,4 \%$ a 30 días y del $14,2 \%$ al año ${ }^{66}$. Desde el punto de vista técnico, el procedi- 
miento valve in valve se asocia con menor tasa de leak paravalvular y de implante de marcapasos definitivo, pero presenta mayor tasa de oclusión coronaria, particularmente en las válvulas en donde las valvas están suturadas por fuera del stent. En tamaño de válvulas pequeñas $(<22 \mathrm{~mm})$, la válvula CoreValve, presentó mayor orificio valvular efectivo comparado con la SAPIEN y consecuentemente un menor gradiente transvalvular debido a la posición supraanular de la válvula ${ }^{66}$. La altura del implante (un implante alto) es importante para mejorar el perfil hemodinámico. La profundidad óptima de implante para CoreValve Evolut es de 0-5 $\mathrm{mm}$, mientras que para la SAPIEN XT es de 0-2 $\mathrm{mm}$. Si bien el éxito del procedimiento es alto, la elección del tamaño y del tipo adecuado de prótesis requiere de equipos experimentados que estén familiarizados con todos los dispositivos y resulta de fundamental importancia la evaluación multidisciplinaria a través del heart team. Además es importante determinar los factores de riesgo para el desarrollo de obstrucción coronaria, dado que su incidencia es mayor en este tipo de procedimientos. Se encuentra disponible en forma gratuita una aplicación móvil (Aortic, UBQO Ltd., Reino Unido) para la identificación fluoroscópica y la descripción de las válvulas con y sin stent. Debido a los resultados favorables en los estudios observacionales, el TAVI ha sido adoptado rápidamente para el tratamiento de las válvulas quirúrgicas biológicas degeneradas para evitar una recirugía en los pacientes de alto riesgo quirúrgico.

\subsection{Riesgo intermedio y bajo riesgo quirúrgico}

Varios estudios han demostrado que los pacientes con menor riesgo quirúrgico tienen mejores resultados que los de mayor riesgo ${ }^{67,68}$, Cuando se compara el TAVI con la cirugía convencional, se observa mejor perfil hemodinámico (menos gradientes, mayor área efectiva y menor tasa de mismatch) con el TAVI, a expensas de mayor tasa de leak paravalvular y requerimiento de marcapasos definitivo. El estudio randomizado NOTION $(\mathrm{n}=280)$ que comparó el TAVI (CoreValve) y la cirugía en pacientes de riesgo intermedio no mostró diferencias en el punto final primario combinado de muerte, $\mathrm{ACV}$ e IAM a 1 año (13,1\% vs. $16.3 \%$ para TAVI y cirugía respectivamente; $p=0,43)^{69}$. La tasa de sangrado mayor fue menor con TAVI, mientras que los pacientes sometidos a cirugía convencional tuvieron menor tasa de requerimiento de marcapasos definitivo, menor leak paravalvular y menos complicaciones vasculares mayores.

El estudio PARTNER 2A ( $\mathrm{n}=2032)$ también comparó el TAVI (SAPIEN XT) con la cirugía en pacientes de riesgo intermedio (STS 5,8\%) sin encontrar diferencias en el punto final primario de muerte y ACV a 2 años $(19 \% \text { vs. } 21 \% \text {, respectivamente })^{70}$. En este estudio el subgrupo de TAVI por vía transfemoral presentó una reducción en el punto final primario comparado con la cirugía. Si bien los pacientes sometidos a TAVI desarrollaron con mayor frecuencia complicaciones vasculares $(7,9$ vs $5 \% ; \mathrm{p}=0,008)$, presentaron menor sangrado que compromete la vida (10 vs. $43 \%$; $\mathrm{p}<0,001)$, injuria renal aguda $(1,3$ vs. $3,1 \% ; \mathrm{p}=0,006)$ y nueva fibrilación auricular (9,1\% vs. $26 \% ; \mathrm{p}<0,001)$. A dos años de seguimiento, el $26 \%$ de los pacientes sometidos a TAVI presentó insuficiencia aórtica paravalvular leve vs. 3,5\% en los pacientes operados, mientras que la tasa de insuficiencia aórtica paravalvular moderada-severa fue de $8 v$ s. 0,6\%. Por último, el estudio no aleatorizado PARTNER 2-S3i $(\mathrm{n}=1078)$ en pacientes de riesgo intermedio (STS 5,3\%) y utilizando la válvula SAPIEN 3 demostró superioridad del TAVI sobre la cirugía convencional en términos de muerte total o ACV en comparación con los pacientes sometidos a cirugía en el PARTNER 2A mediante un score de propensión ${ }^{71}$. Se encuentra en realización el estudio SURTAVI que compara la válvula CoreValve Evolut $\mathrm{R}$ y la cirugía en pacientes de riesgo intermedio, aunque los resultados de los estudios sugieren que, en pacientes de riesgo intermedio, el TAVI es una alternativa a la cirugía convencional, en especial si es por vía transfemoral.

En pacientes de bajo riesgo recientemente han comenzado dos estudios comparativos que evalúan la válvula SAPIEN 3 y CoreValve Evolut R en esta población. En la actualidad, la cirugía de reemplazo valvular aórtica continúa siendo el tratamiento de elección en pacientes de bajo riesgo, debido a que la durabilidad de las válvulas percutáneas no ha sido establecida. cientes de bajo riesgo, debido a que la durabilidad de las válvulas percutáneas no ha sido establecida.

\section{BIBLIOGRAFÍA}

1. Lindroos MR, Kupari M, HeikkiliiJ.Prevalence of Aortic ValveAbnormalities in the Elderly: AnEchocardiographicStudy of a Random Population Sample.J Am Coll Cardiol. 1990;21(5):1220-5.

2. Lax J, Grancelli H, Prezioso H, Sarmiento R, Agatiello C, Avegliano G, et al. Consenso de valvulopatías. Rev Argent Cardiol. 2015;83(2).

3. Lax J. Consenso de Cardiopatías en el Geronte / Versión resumida. Rev Argent Cardiol. 2016;84(3):262-79.

4. Eacts CS, Germany HB, Baro G, Andrew M, Germany B, Bonis M De, et al. Guidelines on the management of valvular heart disease (version 2012): the Joint Task Force on the Management of Valvular Heart Disease of the European Society of Cardiology (ESC) and the European Association for Cardio-Thoracic Surgery (EACTS). Eur Hear J. 2012;33:2451-2496.
5. Otto CM, Guyton RA, Gara PTO, Sorajja P, Thomas JD, Creager MA. 2014 AHA/ ACC Guideline for the Management of Patients With Valvular Heart Disease. J Am Coll Cardiol. 2014;63(22):e57-185.

6. Holmes DR, Mack MJ, Kaul S, Agnihotri A, Alexander KP, Bailey SR, Calhoon JH, Carabello BA, Desai MY, Edwards FH, Francis GS, Gardner TJ, Kappetein AP, Linderbaum JA, Mukherjee D, Otto CM, Ruiz CE, Sacco RL, Smith D, Thomas JD. 2012 ACCF / AATS / SCAl / STS Expert Consensus Document on Transcatheter Aortic Valve Replacement. J Am Coll Cardiol. 2012:59(13):1200-1254

7. WebbJ, Rodés-Cabau J, Fremes S, PibarotP, Ruel M, Ibrahim R, et al. Transcatheter Aortic Valve Implantation: A Canadian Cardiovascular Society Position Statement. Can J Cardiol. 2012;28(5):520-528. 
8. StorteckyS, Schoenenberger AW, Moser A, Wenaweser P. Evaluation of Multidimensional Geriatric Assessment as a Predictor of Mortality and Cardiovascular Events After Transcatheter Aortic Valve Implantation. JACC Cardiovasc Interv. 2012;5(5):489-496.

9. Kamga $M$, Boland B, Cornette P. Impact of frailty scores on outcome of octogenarian patients undergoing transcatheter aortic valve implantation. Acta Cardiol. 2013;68(6):599-606.

10. KodaliSK, Williams MR, Smith CR, Svensson LG, WebbJG,MakkarRR, Fontana GP, Dewey TM, Thourani VH, Pichard AD, Fischbein M, Szeto WY, Lim S, Greason KL, Teirstein PS, Malaisrie SC, Douglas PS, Hahn RT, Whisenant B, Zajarias A, Wang $D$, Akin JJ, Anderson LMPTI. Two-Year Outcomes after Transcatheter or Surgical Aortic-Valve Replacement. NEngl J Med. 2012;366(18):1686-95.

11. Leon MB, Smith CR, MackM, MillerDC, Moses JW, Svensson LG, Tuzcu EM, Webb $J$ G, Fontana GP, MakkarRR, Brown DL, BlockPC, Guyton RA, Pichard AD, Bavaria JE, Herrmann HC, Douglas PS, Petersen JL, Akin JJ, Anderson WN, Wang DPSPTI. Transcatheter aortic-valve implantation for aortic stenosis in patients who cannot undergo surgery. NEngl J Med. 2010;363(17):1597-1607.

12. Makkar RR, Fontana GP, Jilaihawi H, Kapadia S, Pichard AD, Douglas PS, Thourani VH, Babaliaros VC, Webb JG, Herrmann HC, Bavaria JE, Kodali S, Brown DL, Bowers B, Dewey TM, Svensson LG, Tuzcu M, Moses JW, Williams MR, Siegel RJ, Akin JJ, Anderson WN, Poco LMPTI. Transcatheter Aortic-Valve Replacement for Inoperable Severe Aortic Stenosis. N Engl J Med. 2012;366(18):1696-704.

13. Smith CR, Leon MB,MackMJ,MillerDC,Moses JW, Svensson LG, TuzCuEM, Webb JG, Fontana GP,MakkarRR, Williams M, DeweyT, KapadiaS, Babaliaros V, Thourani VH, Corso P, Pichard AD, Bavaria JE, Herrmann HC, Akin JJ, Anderson WN Wang D PSPTI. Transcatheter versus surgical aortic-valve replacement in high-risk patients. N Engl J Med. 2011;364(23):2187-2198.

14. Piazza N, Lange R, Martucci G, Serruys PW. Patient selection for transcatheter aortic valve implantation: Patient risk profile and anatomical. Arch CardiovasC Dis. 2012;105(3):165-173.

15. Piazza N, Hill A, Brockmann G, Opitz A, Mazzitelli D, Elhmidi Y, Ruge H, Bleiziffer S, Lange R. Differences in clinical outcomes based on adherence to valve sizing criteria using 2D echocardiography or 3D computed tomography in patients undergoing transcathether aortic valve implantation. J Am Coll Cardiol. 2012;59(13):E328.

16. Tzikas A, Schultz CJ, Piazza N, Moelker A, Mieghem NM Van, Nuis R, Geuns R Van. Assessment of the Aortic Annulus by Multislice Computed Tomography, Contrast Aortography, and Trans-Thoracic Echocardiography in Patients Referred for Transcatheter Aortic Valve Implantation. Catheter Cardiovasc Interv. 2011;77(6):868-875.

17. Leipsic J, Gurvitch R, Labounty TM, Min JK, WoodD, Johnson M, Ajlan AM, WijesingheN, Webb JG. Multidetector Computed Tomography in Transcatheter Aortic Valve Implantation. JACC Cardiovasc Imaging. 2011;4(4):416-29.

18. Husser O, Rauch S, Endemann DH, Resch M, Nunez J, Bodi V, Hilker M, Schmid C. Impact of Three-Dimensional Transesophageal Echocardiography on Prosthesis Sizing for Transcatheter Aortic Valve Implantation. Catheter Cardiovasc Interv. 2012;80(6):956-963.

19. Cura FA, Albertal M, Padilla L, Nau G, Sztejfman M, Avegliano G, Navia D, TriviM, Ronderos R, Belardi J. Percutaneous Aortic Valve Replacement in High-Risk Patients. Rev Argent Cardiol. 2009;77:203-7.

20. Khatri P, Webb J, Rodés-Cabau J, Fremes S, Ruel M, Lau K, Guo H, Wijeysundera H, Ko D. Annals of Internal Medicine Review Adverse Effects Associated With Transcatheter Aortic Valve Implantation. Ann Intern Med. 2016;158(1):35-46.

21. Blackstone EH, Suri RM, Rajeswaran J, Babaliaros V, Douglas PS, Fearon WF, MiIler DC, Hahn RT, Kapadia S, Kirtane AJ, Kodali SK, Mack M, Szeto WY, Thourani VH, Tuzcu EM, Williams MR, Akin JJ, Leon MB, Svensson LG. Valvular Heart Disease Propensity-Matched Comparisons of Clinical Outcomes After Transapical or Transfemoral Transcatheter Aortic Valve Replacement. Circulation. 2015;131:1989-2000

22. Leon MB, Piazza N, Nikolsky E, Blackstone EH, Cutlip DE, Kappetein AP, KrucoffMW, Mack M, Mehran R, Miller C, Morel M, Petersen J, Popma JJ, Takkenberg JJM, Vahanian A, Es G Van, Vranckx P, Webb JG, Windecker S, Serruys PW. Standardized Endpoint Definitions for Transcatheter Aortic Valve Implantation Clinical Trials A Consensus Report From the Valve Academic Research Consortium. J Am Coll Cardiol. 2011;57(3):253-269.

23. Kappetein AP, Head SJ, Philippe G, Piazza N, Mieghem NM Van, Blackstone EH, Brott TG, Cohen DJ, Cutlip DE, Es G Van, Hahn RT, Kirtane AJ, KrucoffMW, KodaliS, MackMJ, Mehran R, VranckxP,Webb JG, Windecker S, Serruys PW, Leon MB Updated standardized endpoint definitions for transcatheter aortic valve implantation: The Valve Academic Research Consortium-2 consensus document. J Am Coll Cardiol. 2012;60(15):1438-54.
24. Athappan G, Patvardhan E, Tuzcu E, Svensson L, Lemos P, Fraccaro C, et al. Incidence, Predictors, and Outcomes of Aortic Regurgitation After Transcatheter Aortic Valve Replacement. J Am Coll Cardiol. 2013;61 (15):1585-1595.

25. Manoharan G, Walton A, Brecker S, Pasupati S, Blackman DJ, Qiao H, Meredith IT. Treatment of Symptomatic Severe Aortic Stenosis With a Novel Resheathable Supra-Annular Self-Expanding Transcatheter Aortic Valve System. JACC Cardiovasc Interv. 2015:8(10):1359-67.

26. WebbJ, Gerosa G, Lefèvre T, Leipsic J, SpenceM, Thomas M, Thielmann M, Treede $H$, WendlerO, WaltherT.MulticenterEvaluation ofaNext-Generation Balloon-Expandable Transcatheter Aortic Valve.J Am Coll Cardiol. 2014;64(21):2235-2243.

27. Meredith Am I, Walters D, Dumonteil N, Worthley SG, Tchétché D, Manoharan G, Blackman DJ, Lange R, Müller R, Redwood S, Allocco DJ. Transcatheter Aortic Valve ReplacementforSevereSymptomatic Aortic Stenosis Using a Repositionable Valve System. J Am Coll Cardiol. 2014;64(13):1339-8.

28. SinningJ, HammerstinglC, Vasa-nicoteraM, AdenauerV, JosefinaS, Cachiguango L, Scheer A, Hausen S, Sedaghat A, Ghanem A, Müller C, Grube E, Nickenig G, Werner N. Aortic Regurgitation Index Defines Severity of Peri-Prosthetic Regurgitation and Predicts Outcome in Patients After Transcatheter Aortic Valve Implantation. J Am Coll Cardiol. 2012;59(13):1134-1141.

29. Nuis R, Mieghem NM Van, Schultz CI, Tzikas A, Boon RM Van Der, Maugenest A, Cheng J, Piazza N, Domburg RT Van, Serruys PW, Jaegere PP De. Timing and potential mechanisms of new conduction abnormalities during the implantation of the Medtronic CoreValve System in patients with aortic stenosis. Eur Hear J. 2011:32(16):2067-2074

30. Siontis GCM, JüniP, Pilgrim T, StorteckyS, Büllesfeld L, Meier B, WenaweserP, Windecker S. Predictors of Permanent Pacemaker Implantation in Patients With Severe Aortic Stenosis Undergoing TAVR. J Am Coll Cardiol. 2014;64(2):129-140.

31. Guetta V, Goldenberg G, Segev A, Dvir D. Predictors and Course of High-Degree Atrioventricular Block After Transcatheter Aortic Valve Implantation Using the CoreValve Revalving system. Am J Cardiol. 2010;108(11):1600-1605.

32. PereiraMM, MinhaS, Alarcon RE, BakerN, Ota H, OmarAF, Pendyala L, Loh J, Rodriguez-weisson JF, Torguson R, Chen F, Ben-dor I, Okubagzi P, Suddath W, Satler L, Pichard A, Waksman R. The Impact of Permanent Pacemaker Implantation Following Transcatheter Aortic Valve Replacement on Outcome. J Am Coll Cardiol. 2016;63(12):A1735.

33. GénéreuxP,WebbJG, Svensson LG, Kodali SK, Satler LF, Fearon WF, et al. Vascular Complications After TranscatheterAortic ValveReplacement(PlacementofAoRTic TraNscathetER Valve) Trial. J Am Coll Cardiol. 2012;60(12):1043-52.

34. Mieghem NM Van, TchetcheD, Chieffo A, Dumonteil N, Messika-zeitoun D, Boon RMA Van Der, Vahdat O, Buchanan GL, Marcheix B, HimbertD, Serruys PW, Fajadet J, Colombo A, Carrié D, Vahanian A. Incidence, Predictors, and Implications of Access Site Complications With Transfemoral Transcatheter Aortic Valve Implantation. Am J Cardiol. 2012;110(9):1361-1367.

35. Hayashida K, Lefèvre T, Chevalier B, Hovasse T, Romano M, Garot P, Mylotte D. Transfemoral Aortic Valve Implantation New Criteria to Predict Vascular Complications. JACC Cardiovasc Interv. 2011;4(8):851-858.

36. Stortecky S, WenaweserP, Diehm N, Pilgrim T, HuberC, RosskopfAB, KhattabAA, Buellesfeld L, Gloekler S. Percutaneous Management of Vascular Complications in Patients Undergoing Transcatheter Aortic Valve Implantation. JACC Cardiovasc Interv. 2012;5(5):515-524.

37. Toggweiler S, Leipsic J, Binder RK, Freeman M, Barbanti M, Heijmen RH, Wood $D A$, Webb J G. Management of Vascular Access in Transcatheter Aortic Valve Replacement. JACC Cardiovasc Interv. 2013;6(8):767-76.

38. Ludman PF, MoatN, BelderMADe, Blackman DJ, Duncan A, Banya W, Maccarthy PA, Cunningham D, Wendler O, Marlee D, Hildick-smith D, Young CP, Kovac J, Uren NG, Spyt T, Trivedi U. Transcatheter aortic valve implantation in the United Kingdom: temporal trends, predictors of outcome, and 6-year follow-up: a report from the UKTranscatheter Aortic Valve Implantation (TAVI) Registry, 2007 to 2012. Circulation. 2015;131(13):1181-90.

39. Auffret V, Regueiro A, Del Trigo M, Abdul-Jawad Altisent O, Campelo-Parada F, Chiche O, PuriR, Rodés-CabauJ. Predictors of Early Cerebrovascular Events in Patients With Aortic Stenosis Undergoing Transcatheter Aortic Valve Replacement. J Am Coll Cardiol. 2016;68(7):673-84.

40. Van Belle, EricHengstenberg C, Lefevre T, Kupatt C, Debry N, Husser O, et al. Cerebral Embolism During Transcatheter Aortic Valve Replacement: The BRAVO-3 MRI Study. J Am Coll Cardiol. 2016;68(6):589-99.

41. Haussig S, Mangner N, Dwyer MG, LehmkuhI L, Lücke C, Woitek F, Holzhey DM, Mohr FW, Gutberlet M, Zivadinov R, Schuler G, Linke A. Effect of a Cerebral Protection Device on Brain Lesions Following Transcatheter Aortic Valve Implantation in Patients With Severe Aortic Stenosis The CLEAN-TAVI Randomized Clinical Trial. JAMA. 2016;316(6):592-601. 
42. Kapadia SR, KodaliS, MakkarR, Mehran R, LazarRM, ZivadinovR, DwyerMG, Jilaihawi H, Virmani R, Anwaruddin S, Thourani VH, NazifT, MangnerN, WoitekF, Krishnaswamy A, MickS, Chakravarty T, Nakamura M, Mccabe JM, Satler L, Zajarias A, Szeto WY, Svensson L, Alu MC, White RM, Kraemer C, Parhizgar A, Leon MB, Linke A. Cerebral Embolic Protection During Transcatheter Aortic Valve Replacement. J Am Coll Cardiol. 2016.

43. Ewe SH, Muratori M, Delgado V, Pepi M, Tamborini G, Fusini L, Klautz RJM, Gripari P, Bax JJ, Fusari M, Schalij MJ, Marsan NA. Hemodynamic and Clinical Impact of Prosthesis - Patient Mismatch After Transcatheter Aortic Valve Implantation. J Am Coll Cardiol. 2011;58(18):1910-1918.

44. Dangas G, Lefèvre T, KupattC, TchetcheD, Schäfer U, Dumonteil N, WebbJG, Colombo A, Windecker S, Berg M, Hildick-smith D, Mehran R, Boekstegers P, Linke A, Tron C, Belle E Van, Asgar AW, Fach A, Jeger R, Sardella G, Hink HU, Husser O, Grube E, Deliargyris EN, Lechthaler I, Bernstein D, Wijngaard P, Anthopoulos P.Bivalirudin Versus Heparin Anticoagulation in Transcatheter Aortic Valve Replacement: The Randomized BRAVO-3 Trial. J Am Coll Cardiol. 2015;66(25):2860-8.

45. Ussia GP, ScarabelliM, MulèM, BarbantiM, SarkarK, Cammalleri V, ImmèS, ArutaP, Pistritto AM, Gulino S, DesteW. Dual Antiplatelet Therapy Versus Aspirin Alonein Patients Undergoing Transcatheter Aortic Valve Implantation. Am J Cardiol. 2010;108(12):1772-1776

46. StabileE, Pucciarelli A, Cota L, Sorropago G, Tesorio T, Salemme L, PopusoiG, Ambrosini V, Cioppa A, Agrusta M, Catapano D, Moscariello C, Trimarco B, Esposito G, Rubino P. SAT-TAVI ( single antiplatelet therapy for TAVI) study: A pilot randomized study comparing double to single antiplatelet therapy for transcatheter aortic valve implantation. Int J Cardiol. 2014;174(3):624-627.

47. Poliacikova P, Cockburn J, deBelderA, TrivediU, Hildick-Smith D. Antiplatelet and antithrombotic treatment after transcatheter aortic valve implantation - comparison of regimes. J Invasive Cardiol. 2013;25(10):544-8.

48. Durand E, Blanchard D, Chassaing S, Gilard M, Laskar M, Borz B, Lafont A, Barbey C, Godin M, Tron C, Zegdi R, Chatel D, Page O Le, Litzler P, Bessou J, Danchin $N$, Cribier A, EltchaninoffH. Comparison of Two Antiplatelet Therapy Strategies in Patients Undergoing Transcatheter Aortic Valve Implantation. Am J Cardiol. 2014;113(2):355-360.

49. WhitlockRP, Eikelboom JW. Prevention of thromboembolic events after bioprosthetic aortic valve replacement. J Am Coll Cardiol. 2012;60(11):978-80

50. De Marchena E, Mesa J, Pomenti S, Marin Y Kall C, Marincic X, Yahagi K, Ladich E, Kutys R, Aga Y. Thrombus Formation Following Transcatheter Aortic ValveReplacement. JACC Cardiovasc Interv. 2015;8(5):728-739.

51. Makkar R, Fontana G, Jilaihawi H, Chakravarty T, Kofoed K, de Backer O, Asch F, RuizC, Olsen N, Trento A, Friedman J, Berman D, Cheng W, KashifM, Jelnin V, Kliger C, Guo H, Pichard A, Weissman N, Kapadia S, Manasse E, Bhatt D, Leon M, Søndergaard L. Possible Subclinical Leaflet Thrombosis in Bioprosthetic Aortic Valves. NEngl J Med. 2015;373(21):2015-2024.

52. Hansson N, Grove E, Andersen H, Leipsic J, Mathiassen ON, Jensen JM. Transcatheter Aortic Heart Valve Thrombosis: Incidence, Predisposing Factors, and Clinical Implications. J Am Coll Cardiol. 2016.

53. Moris C, Pascuall, Avanzas P. Will TAVI Be the Standard of Care in the Treatment ofAortic Stenosis? Rev Esp Cardiol. 2016.

54. Go A, Hylek EM, Phillips KA, Henault LE, Selby J V, Singer DE. Prevalence of diagnosed atrial fibrillation in adults: national implications for rhythm management and stroke prevention: the AnTicoagulation and Risk Factors in Atrial Fibrillation (ATRIA) Study. JAMA. 2016;285(18):2370-2375.

55. Mario C Di, EltchaninoffH, Moat N, Goicolea J, Ussia GP, Kala P, Wenaweser P, Zembala M, Nickenig G, Barrero EA, Snow T, lung B. The 2011-12 pilot European Sentinel Registry of Transcatheter Aortic Valve Implantation: in-hospital results in 4,571 patients. Eurolntervention. 2013;8(12):1362-71.

56. Miyasaka Y, Barnes M, Bailey K, Cha SS, Gersh BJ, Hil DP, Seward JB, Tsang TSM. Mortality Trends in Patients Diagnosed With FirstAtrial Fibrillation. J Am Coll Cardiol. 2007:49(9):986-92.

57. Mariscalco G, Klersy C, Zanobini M, Banach M, Ferrarese S, Borsani P, Cantore C, Biglioli P, Sala A. Atrial fibrillation after isolated coronary surgery affects late survival. Circulation. 2008:118(16):1612-8.
58. Sannino A, Gargiulo G, Schiattarella GG, Perrino C, Stabile E, Losi M, Galderisi M. A meta-analysis of the impact of pre-existing and new-onset atrial fibrillation on clinical outcomes in patients undergoing transcatheter aortic valve implantation. Eurolntervention. 2016;12(8):e1047-56.

59. Arsalan $M$, Walther T. Durability of prostheses for transcatheter aortic valve implantation. Nat Rev Cardiol. 2016;13(6):360-7.

60. Aranzulla T, De Benedictis M, Scrocca I, Mabritto B, Pizzuti A, Meliga E, RovereM, ConteM. Efficacy of a 1-yearfollow-up program conducted in a tertiary carecentre following percutaneous aortic valve implantation: the TAVI follow-up program. JAm Coll Cardiol. 2015;66(15_S).

61. Roberts W. The congenitally bicuspid aortic valve. A study of 85 autopsy cases. Am J Cardiol. 1970;26(1):72-83.

62. YousefA, SimardT, Webb J, Rodés-cabau J, Costopoulos C, Kochman J, Hernández-garcia JM, Chiam PTL, Welsh RC, Wijeysundera HC, García E, Ribeiro HB, Latib A, HuczekZ, Shanks M, Testa L, FarkouhME, DvirD, Velianou JL, LamB, Pourdjabbar A, Glover C, Hibbert B, Labinaz M. Transcatheter aortic valve implantation in patients with bicuspid aortic valve: A patient level multi-center analysis. Int J Cardiol. 2015:189:282-288.

63. Bauer T, Linke A, Sievert H, KahlertP,HambrechtR, Nickenig G, et al. Comparison of the Effectiveness of Transcatheter Aortic Valve Implantation in Patients With Stenotic Bicuspid Versus Tricuspid Aortic Valves (from the German TAVI Registry). Am J Cardiol. 2014;113(3):518-521.

64. Yoon S, Lefèvre T, Ahn J, Perlman GY, Dvir D, Latib A, Barbanti M, DeuschIF, BackerODe, BlankeP, Modine T, Pache G, Neumann F, Ruile P, Arai T, Ohno Y, Kaneko H, Tay E, Schofer N, Holy EW, LukNHV, Yong G, Lu Q, Kong WKF, Hon J, Kao H, Lee M, Yin W, ParkD, Kang S, Lee S, Kim Y, Lee CW, Park S, Kim H, Butter C, Khalique OK, Schaefer U, Nietlispach F, Kodali SK, Leon MB, Ye J, Chevalier B, Leipsic J, Delgado V, Bax JJ, Tamburino C, Colombo A, Søndergaard L, Webb JG, ParkS. Transcatheter Aortic Valve Replacement With Early-and New-Generation Devices in Bicuspid Aortic Valve Stenosis. J Am Coll Cardiol. 2016;68(11):1195-1205.

65. Franzone A, Piccolo R, Siontis GCM, Lanz J, Stortecky S, Praz F, Roost E, Mpp RV, WindeckerS, Pilgrim T. TranscatheterAortic Valve Replacementforthe Treatment of Pure Native Aortic\&nbsp;Valve Regurgitation. JACC Cardiovasc Interv. 2016.

66. Dvir D, Webb J, Bleiziffer S, Pasic M, Waksman R, Kodali S, Barbanti M, Latib A SchaeferU, Rodés-Cabau J, TreedeH, PiazzaN, Hildick-Smith D, HimbertD, Walther T, Hengstenberg C, Nissen H, Bekeredjian R, Presbitero P, Ferrari E, Segev A, de Weger A, Windecker S, Moat N, Napodano M, Wilbring M, Cerillo A, Brecker S, Tchetche D, Lefèvre T, De Marco F, Fiorina C, Petronio A, Teles R, Testa L, Laborde J, Leon M, Kornowski R, Investigators. V-VIDR. Transcatheter Aortic Valve Implantation in Failed Bioprosthetic Surgical Valves. JAMA. 2014;312(2):162-170.

67. Wenaweser P, StorteckyS, Schwander S, Heg D, Huber C, Pilgrim T, Gloekler S, SuIlivan CJO, Carrel T, Windecker S, Meier B, Ju P. Clinical outcomes of patients with estimated low or intermediate surgical risk undergoing transcatheter aortic valve implantation. Eur Hear J. 2013;34(25):1894-905.

68. Piazza N, Kalesan B, Mieghem N Van. A 3-Center Comparison of 1-Year Mortality Outcomes Between Transcatheter Aortic Valve Implantation and Surgical Aortic Valve Replacement on the Basis of Propensity Score Matching Among Intermediate-Risk Surgical Patients. JACC Cardiovasc Interv. 2013;6(5):443-451.

69. Nissen H, Kjeldsen J, Petursson P, Chang Y. Transcatheter Versus Surgical Aortic Valve Replacement in Patients With Severe Aortic Valve Stenosis. J Am Coll Cardiol. 2015;65(20):2184-94.

70. Leon M, Smith C, MackM, MakkarR, Svensson LG, PhD, KodaliSK, Thourani VH, TuzcuEM, Pichard AD, Kapadia S, Dewey T, Babaliaros V, Szeto WY, Williams MR, Kereiakes D, Zajarias A, Greason KL, Whisenant BK, Hodson RW, Moses JW, Trento A, Brown DL, Fearon WF, Pibarot P, Ph D, Hahn RT, Jaber WA, Anderson WN, Ph D, Alu MC, Webb JG, Investigators P. Transcatheter or Surgical Aortic-Valve Replacement in Intermediate-Risk Patients. NEngl J Med. 2016;374:1609-1620.

71. ThouraniVH, KodaliS, MakkarRR, Herrmann HC, Williams M, Babaliaros V,SmaIling R, Lim S, Webb JG, Moses JW, MackMJ, MillerDC, Smith CR, Alu MC, Parvataneni $R$, Jr RBDA, Leon MB. Transcatheter aortic valve replacement versus surgical valve replacement in intermediate-risk patients: a propensity score analysis. Lancet. 2016;387(10034):2218-25. 